\title{
Au-delà de la collecte des données dans les projets de sciences citoyennes : ouvrir le champ de l'analyse et de l'interprétation des données aux citoyens.
}

\author{
Beyond the collection of data in citizen science projects. Opening the \\ field of data analysis and interpretation to citizens.
}

\author{
Sylvie Blangy ${ }^{1}$, Valentin Lhoste ${ }^{1,2}$, Céline Arnal ${ }^{3}$, ,Jennifer Carre ${ }^{4}$, Anaïs Chapot $^{1}$, Isabelle \\ Chuine $^{1}$, Gaëlle Darmon ${ }^{1}$, Alexis Joly ${ }^{6}$, Pascal Monestiez ${ }^{5}$, Pierre Bonnet ${ }^{2,5}$ \\ ${ }^{1}$ Centre d'Ecologie Fonctionnelle et Evolutive (CEFE), UMR 5175, CNRS, Montpellier, France. \\ sylvie.blangy@cefe.cnrs.fr, isabelle.chuine@cefe.cnrs.fr, chapotanais@outlook.fr, gaelle.darmon@cefe.cnrs.fr \\ ${ }^{2}$ Centre de coopération internationale en recherche agronomique pour le développement (CIRAD), UMR AMAP, \\ Montpellier, France, Ihoste.v@hotmail.fr, pierre.bonnet@cirad.fr \\ ${ }^{3}$ Cybelle Planète, Villeneuve les Maguelone, France, celine.arnal@cybelle-planete.org \\ ${ }^{4}$ Tela Botanica, Montpellier, France, jenni.carre@gmail.com \\ ${ }^{5}$ Unité mixte de botanique et Modélisation de l'Architecture des Plantes et des végétations (AMAP), Université de \\ Montpellier, CIRAD, CNRS, INRA, IRD, Montpellier, France, pierre.bonnet@cirad.fr \\ ${ }^{6}$ INRIA (signification) Sophia-Antipolis - ZENITH team, LIRMM, Montpellier, France, alexis.joly@inria.fr \\ ${ }^{7}$ BioSP, INRA, Site Agroparc, Avignon, pascal.monestiez@inra.fr
}

RÉSUMÉ. Les sciences participatives et citoyennes ont pris depuis une dizaine d'années un essor sans précédent, dans un grand nombre de disciplines, s'appuyant sur une large mobilisation citoyenne, le développement du numérique et de nouvelles formes d'interaction science-société. Pour les questions d'écologie et d'environnement, le citoyen est souvent cantonné dans la production de données. Notre objectif est d'étudier les innovations récentes, dans des programmes dédiés à l'écologie et l'environnement, qui impliquent plus le citoyen dans l'analyse, l'interprétation ou la construction de nouvelles questions à partir des données récoltées, et d'en tirer des leçons pour l'avenir. Au travers de l'étude d'une trentaine d'exemples, nous analysons les différents types d'implication citoyenne, les facteurs favorisants, les outils mis en œuvre. Nous en déduisons une typologie des projets de sciences citoyennes au regard de leur capacité à impliquer les participants dans l'analyse et l'interprétation. Nous concluons par quelques pistes de développement pour les coordinateurs et gestionnaires de programmes de sciences participatives.

ABSTRACT. Over the last decade, citizen science has experienced an unprecedented expansion into a large number of disciplines, due to increased public participation, the development of a variety of digital applications, and the creation of new and innovative interactions between science and society. In the fields of biodiversity and the environment, the role of citizens is often limited to data collection. The objective of this study was to investigate existing programs employing recent innovations that involve members of the public in the analysis, interpretation and formulation of new questions using collected data in order to draw lessons for the future. In the analysis, 30 case studies were examined to determine the different types of civic involvement, the factors that contributed to this involvement, and the types of tools used. This resulted in a typology of citizen science projects categorized by their ability to involve participants in data analysis and interpretation. The study concludes with different options for coordinators and project managers of citizen science programs.

MOTS-CLÉS. Recherche Action Participative, Science Citoyenne, Analyse de données, interprétation, Co-construction, Qualité et Enrichissement des données, Intelligence Collective.

KEYWORDS. Participatory Action Research, Citizen Sciences, Data analysis, Interpretation, Co-construction, Quality and Enrichment of the data, Collective Intelligence.

\section{Introduction}

Les sciences participatives (ou citoyennes) ont pris depuis une dizaine d'années un essor sans précédent, dans un très grand nombre de disciplines [HOU 16], [KUL 16]. Leur place reste modeste en termes de production scientifique mais leur développement s'est accéléré ces dernières années et leur 
impact sociétal a fortement augmenté. La France occupe actuellement le 7e rang mondial et le 3e rang européen des pays publiant sur le sujet [HOU 16].

Ces nouvelles démarches de recherche s'appuient sur (i) une large mobilisation citoyenne, (ii) l'utilisation de nouveaux outils numériques, (iii) une nouvelle forme d'interaction entre la société civile et la communauté scientifique à l'aide de moyens habiles et d'une ingénierie de la participation basés sur une démarche de Recherche Action Participative (RAP) ${ }^{1}$ [CHE 13]. Ce troisième point prend des formes très variées selon les thématiques de recherche, la dimension des collectifs et des équipements mobilisés. Dans le cadre des recherches sur l'évolution de l'environnement, notamment en rapport aux conséquences du changement climatique, de nombreux projets positionnent essentiellement la contribution des citoyens dans la production d'observations de terrain, visant à étendre la prospection du territoire par le relevé de paramètres environnementaux (température, hygrométrie, salinité, luminosité, floraison...) ou d'occurrences d'organismes vivants. La société civile a cependant des questions et des attentes spécifiques qui nécessitent de l'impliquer au-delà de la collecte de données de terrain. L'implication des citoyens dans toutes les étapes des programmes de recherche a beaucoup d'avantages, autant pour les citoyens que les chercheurs. Pour ces derniers, elle est bénéfique afin de mieux s'intégrer auprès de la société citoyenne, mieux comprendre les acteurs concernés ou mieux engager ces derniers, par exemple.

Nous proposons dans cet article de présenter les innovations récentes développées par des initiatives de sciences citoyennes, qui visent à positionner la contribution citoyenne dans d'autres champs d'actions que la seule production d'observations, notamment dans le développement de nouvelles questions de recherche, et /ou dans l'analyse de données collectées / structurées à des fins de recherche. Ce travail s'appuie sur l'analyse de plusieurs formes de contributions citoyennes dans des programmes internationaux de domaines et régions variés. Nous avons ainsi caractérisé près d'une trentaine de projets sélectionnés au regard de la diversité des approches qu'ils proposent. Ces projets s'appuyant sur des outils et des approches numériques, ont été sélectionnés et évalués en fonction d'une série de critères (types de données, visualisation des données, modalités d'analyse des données...) définis au cours d'une démarche itérative. Leur évaluation permet de réaliser une typologie des projets de sciences citoyennes au regard de leur capacité à offrir aux participants un cadre d'expression en terme d'analyse, d'interprétation ou de diffusion des données.

Les apports provenant de cette exploration sont précieux pour penser le développement de projet de sciences citoyennes dans une perspective autorisant les participants à agir autrement qu'en collectant des données. Dans la section qui suit nous ouvrons la discussion sur les limites actuelles et le potentiel des démarches présentées, en particulier l'analyse des freins (techniques, compétences nécessaires, réticence des scientifiques), et l'analyse des solutions possibles. Nous conclurons par des pistes potentielles d'évolution pour les coordonnateurs et gestionnaires de projet de Sciences Participatives (SP) qui souhaitent développer des partenariats avec les citoyens et trouver d'autres formes d'échanges et d'implication citoyenne, en aval ou en amont de la collecte proprement dite.

\section{Vers une définition consensuelle des sciences citoyennes : la PPSR}

Force est de constater que la signification du terme «Sciences Citoyennes » n'est pas immédiate. Et pour cause, depuis au moins 20 ans les variations sur la thématique de l'implication des citoyens dans la recherche scientifique sont nombreuses. En 1996, Bonney et Irwin employaient déjà le terme de « citizen science » selon des approches différentes. Bonney l'introduisit aux Etats-Unis dans le champ de l'écologie et de la conservation pour décrire des démarches de collecte de données à grande échelle [BON 96]. Irwin (1995) en fit état en Europe dans le champ des sciences sociales pour décrire une philosophie de l'engagement dans les discours scientifiques et les conduites politiques [IRW 95]. Dès lors, de nombreuses formulations sont apparues, laissant entendre de légères variations autour de ce

${ }^{1}$ https://www.participatoryactionresearch.net 
terme. Chez les anglophones, les termes suivants sont utilisés : «volunteer biological monitoring », «community science », «community-based monitoring Chez les francophones, ce sont les termes de «sciences citoyennes», "sciences participatives», «recherche action participative», "recherche action en partenariat» qui sont le plus souvent mentionnés.

La "Citizen Science Association" $\left(\mathrm{CSA}^{2}\right)$ s'impose de plus en plus comme le cadre de référence en termes de sciences citoyennes au niveau international. Elle regroupe plus de mille projets, impliquant près d'un million de participants pour un total de 4000 membres issus de 80 pays. L'association reçoit un appui financier et technique de la part de plusieurs organisations partenaires telles que Cornell Lab of Ornitology, National Geographic, le Muséum d'Histoire Naturelle de Londres ainsi que celui de Los Angeles. Les membres constituants le comité de direction peuvent provenir de certaines de ces organisations mais sont également des universitaires issus de différents instituts de recherche. Il est important de noter cependant, qu'aucun citoyen ne fait partie de ce comité de direction.

La CSA a adopté la définition suivante : "un travail scientifique entrepris par les membres du public, souvent en collaboration avec ou sous la direction de scientifiques professionnels et d'institutions scientifiques ${ }^{3}$. » ([CSA 2017a] ; [CSA 2017b]).. Cette définition, nous dit l'association, provient du «Dictionnaire Anglais d'Oxford » (Oxford English Dictionary, OED) qui l'a récemment intégrée. Cependant, la définition qui est donnée par l'OED à ce jour (juillet 2017) est différente de celle citée sur le site de la CSA. Le site de l'OED définit aujourd'hui le terme de "sciences citoyennes » comme «la collecte et l'analyse de données en relation avec le monde naturel par les membres du public, généralement faisant partie d'un projet collaboratif avec les scientifiques professionnels ${ }^{4} \gg[\mathrm{OED} 2017]$.

Il s'agit probablement d'une mise à jour car d'autre sources utilisent toujours la première version de la définition de CSA [HAK 14] ; [CSA 17a]; [ZOO 14]), néanmoins la différence mérite notre attention. Effectivement la définition reprise notamment par la CSA, possède un élément supplémentaire à savoir que les sciences citoyennes peuvent être autant «en collaboration avec» les scientifiques que «sous la direction de » ces mêmes scientifiques. Alors que la définition actuellement en vigueur sur le site de l'OED ne fait état que d'un travail de "collaboration avec les scientifiques». La disparition de l'approche "top-down" "sous la direction de » dans la définition actuelle de l'OED est intéressante. Cela témoigne du clivage, au sein des sciences citoyennes, entre les approches participatives dirigées et les approches participatives en collaboration avec les chercheurs [LHO].

En 2008, le «Center for the Advancement of Informal Science Education» (CAISE) américain a soutenu un groupe de recherche pour définir le périmètre et l'impact sur le plan éducatif de différents modèles de sciences citoyennes [BON 09b] (tableau 1). Ce groupe, rassemblant des praticiens et chercheurs de différents domaines, a publié un rapport en 2009 pour répondre à cette demande [BON 09a] et un article en 2012 [SHI 12] complétant les idées développées dans le rapport de 2009a. Cet article fait le constat, à propos de l'engagement public dans les sciences, qu'il existe « une confusion parmi les terminologies existantes, compliquant la communication entre les différents projets » [SHI 12] et parle, en reprenant les termes de Rowe et Frewer [ROW 05], d'une "nomenclature incertaine et contradictoire ».

Face à ce constat et dans le souci de formuler des termes distincts et au besoin de partager une appellation commune à travers les différents champs disciplinaires, le groupe de recherche a proposé le

\footnotetext{
${ }^{2}$ http://citizenscience.org/

${ }^{3}$ Traduit de : "scientific work undertaken by members of the general public, often in collaboration with or under the direction of professional scientists and scientific institutions"

${ }^{4}$ Traduit de: "The collection and analysis of data relating to the natural world by members of the general public, typically as part of a collaborative project with professional scientists"
} 
terme de «Participation Publique à la Recherche Scientifique »(PPSR). Les auteurs définissent la PPSR comme « une collaboration intentionnelle dans laquelle des membres du public s'engagent dans un processus de recherche pour générer de nouvelles connaissances basées sur la réflexion scientifique » [SHI 12]. Différents modèles de PPSR ont ainsi pu être définis. La différence majeure entre ces modèles et les définitions vues précédemment réside dans l'introduction d'une notion de degré d'implication aux différentes étapes de la démarche scientifique. Cela implique, que d'un projet à un autre, les participants peuvent être impliqués de manière très différente et ne pas avoir les mêmes rôles. Ce sont ces aspects du degré d'implication et du rôle joué par le citoyen que nous chercherons à préciser dans les parties suivantes.

\section{La croissance et les caractéristiques de la participation dans la recherche scientifique}

\subsection{Impact bibliométrique}

Nous commencerons d'abord par contextualiser les sciences citoyennes et plus particulièrement la Participation Publique aux Recherches Scientifiques (PPRS) au cours des 35 dernières années. Différents rapports ont proposé un état des lieux de la place des sciences participatives au sein de la recherche au niveau mondial. Ainsi le rapport de Houllier et Merilhou-Goudard (2016) [HOU 16] ainsi que celui de la Fondation Sciences Citoyennes [STO 12] rendent compte d'une croissance exponentielle des publications dans le domaine des sciences participatives (figure 1) et utilisant le terme «participation» (figure 2). Il apparaît donc que depuis plus de trente ans environ les sciences participatives ou la notion de participation des citoyens à la recherche scientifique a considérablement augmenté. Le développement exponentiel des technologies de l'information et de la communication, contribuant à celui de la participation dans les sciences, est très certainement une des explications notables de cette croissance. Pour autant, nous avions commencé à l'évoquer, la nature de la participation et l'implication des participants à la recherche peut varier en fonction des modèles préconisés par la notion de PPSR. Aussi, nous chercherons à préciser autant que possible les niveaux de participation pour lesquels il est possible de s'impliquer, notamment au sein des projets proposant une participation du public.

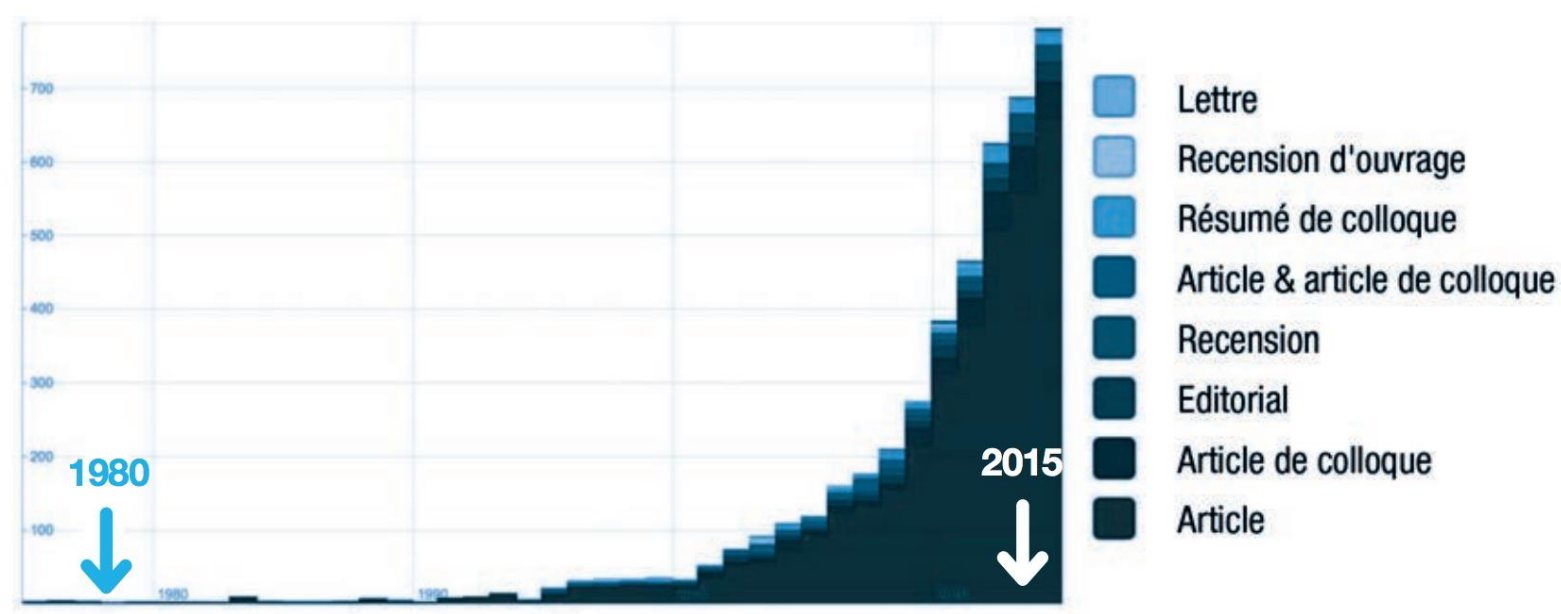

Figure 1. Évolution du nombre de publications en sciences participatives [HOU 16] 


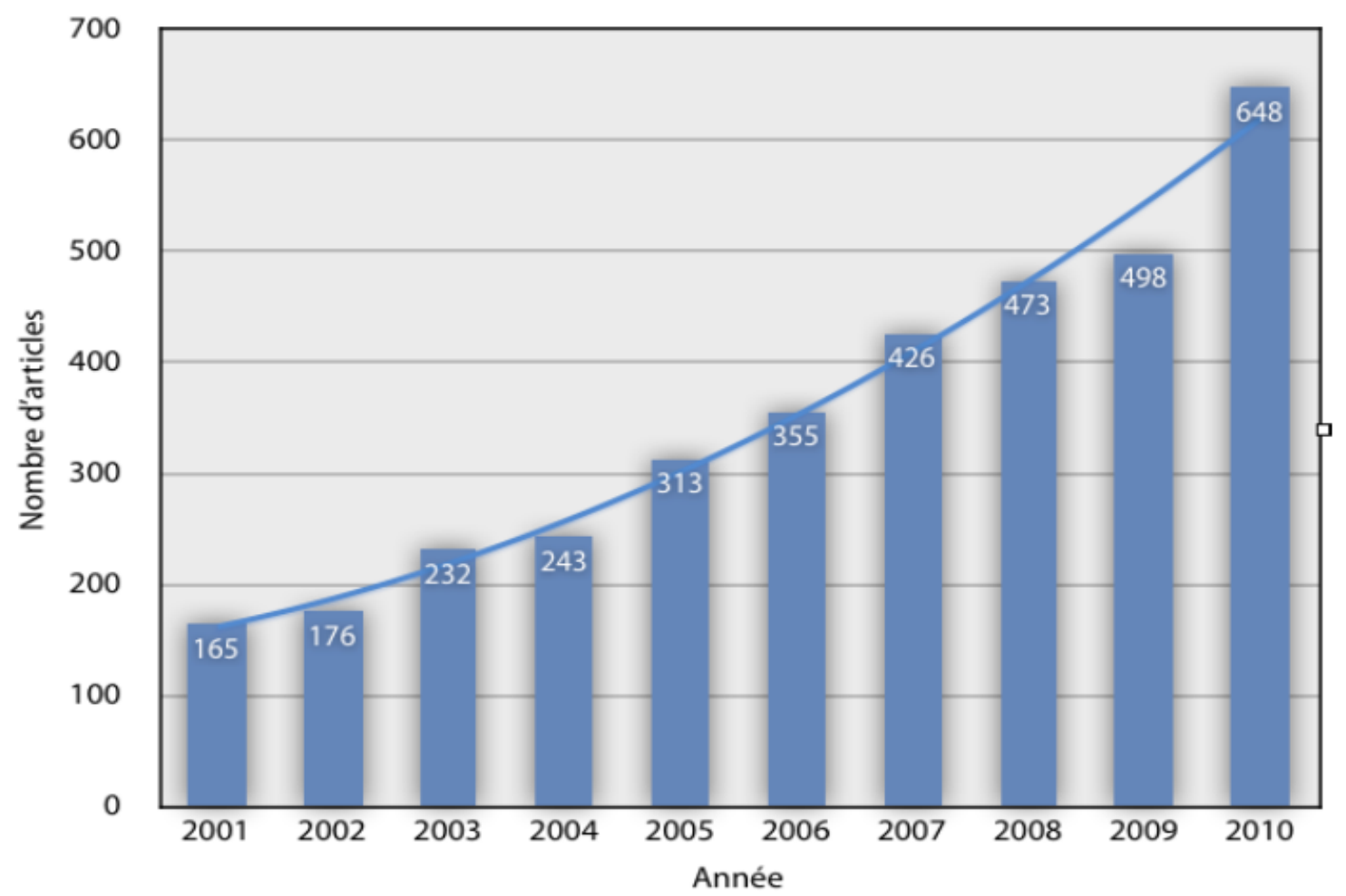

Figure 2. Analyse scientométrique - Mot-clé "Participatory" : Nombre de Publications (2001-2010) " [STO 12]

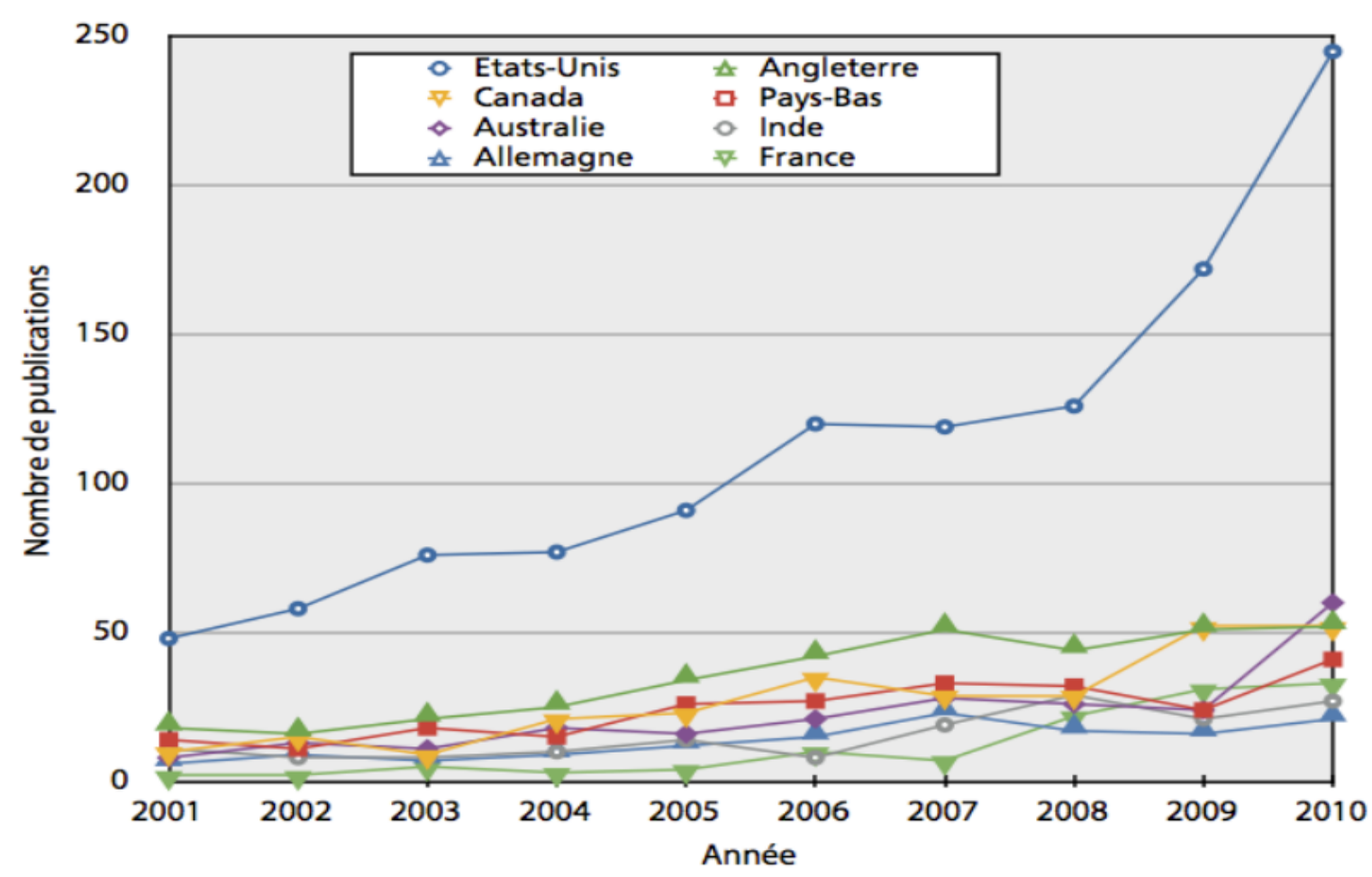

Figure 3. Analyse scientométrique - Mot-clé "Participatory" : Nombre de Publications par pays (2001-2010). $[\mathrm{STO} 12]^{5}$

Plusieurs pays jouent un rôle prédominant dans cette croissance de la participation (figure 3). La participation de la France est mineure par rapport à celle des Etats-Unis, du Canada, de l'Angleterre ou

\footnotetext{
${ }^{5}$ Pays publiant le plus ainsi que les sujets ou disciplines (au sens de la nomenclature de la base de données « Web of science ») dans lesquels ces articles ont publiés 
de l'Australie. Notre travail s'est orienté par conséquent vers les pays d'Amérique du Nord et nous avons privilégié les sites qui avaient leur siège social dans ces pays.

\begin{tabular}{|c|c|c|c|}
\hline Step in Scientific Process & $\begin{array}{l}\text { Steps included } \\
\text { in Contributory } \\
\text { Projects }\end{array}$ & $\begin{array}{l}\text { Steps included } \\
\text { in Collaborative } \\
\text { Projects }\end{array}$ & $\begin{array}{l}\text { Steps included } \\
\text { in Co-created } \\
\text { Projects }\end{array}$ \\
\hline $\begin{array}{l}\text { Choose or define } \\
\text { question(s) for study }\end{array}$ & & & $\mathrm{X}$ \\
\hline $\begin{array}{l}\text { Gather information and } \\
\text { resources }\end{array}$ & & & $\mathrm{x}$ \\
\hline $\begin{array}{l}\text { Develop explanations } \\
\text { (hypotheses) }\end{array}$ & & & $\mathrm{X}$ \\
\hline $\begin{array}{l}\text { Design data collection } \\
\text { methodologies }\end{array}$ & & $(X)$ & $\mathrm{X}$ \\
\hline $\begin{array}{l}\text { Collect samples and/or } \\
\text { record data }\end{array}$ & $x$ & $\mathrm{x}$ & $\mathrm{X}$ \\
\hline Analyze samples & & $x$ & $\mathrm{X}$ \\
\hline Analyze data & $(X)$ & $\mathrm{X}$ & $x$ \\
\hline $\begin{array}{l}\text { Interpret data and draw } \\
\text { conclusions }\end{array}$ & & $(X)$ & $\mathrm{X}$ \\
\hline $\begin{array}{l}\text { Disseminate conclusions/ } \\
\text { translate results into action }\end{array}$ & $(X)$ & $(X)$ & $x$ \\
\hline $\begin{array}{l}\text { Discuss results and ask } \\
\text { new questions }\end{array}$ & & & $\mathrm{x}$ \\
\hline
\end{tabular}

$\mathrm{X}=$ public included in step; $(\mathrm{X})=$ public sometimes included in step

Tableau 1. Modèles de la Participation Publique aux Recherches Scientifiques [BON 09a]

\subsection{Intérêt des sciences participatives du point de vue des citoyens}

La participation publique aux recherches scientifiques (PPRS) paraît effectivement très intéressante du point de vue des chercheurs, notamment avec une collecte importante de données par les participants. Les données fournies permettent de couvrir des territoires plus étendus et des périodes de temps plus longues. Le volant de données s'étoffe et les travaux des chercheurs y gagnent en crédibilité. Cependant, il est important que les participants y trouvent aussi un intérêt. Il est fort probable que cela soit cet intérêt qui soit à l'origine de la forte expansion de la participation citoyenne au sein des projets de recherche. Pour illustrer notre propos, nous nous baserons sur les résultats d'une enquête Ipsos de Mai 2016 [MER 16] répertoriés en annexe 1.

Cette enquête ainsi que les travaux de Van der Leew (VAN 2016) confirment que les sciences participatives sont un moyen de renforcer les liens entre le grand public et la recherche et de faire progresser la science.

$87 \%$ des répondants pensent qu'une implication plus forte du grand public dans les travaux de recherche scientifique pourrait permettre aux citoyens de mieux comprendre les enjeux de société des avancées scientifiques et techniques, et de renforcer le lien entre les chercheurs et les citoyens. Cela présuppose que les citoyens comprennent plus ou moins bien les enjeux de société dans la recherche. De fait, les citoyens ne se font plus leur propre idée des enjeux relatifs à la science, ils se font l'idée que les institutions scientifiques et politiques relaient. Les citoyens pourraient effectivement trouver dans les sciences participatives un moyen de se réapproprier la résolution des enjeux sociétaux de notre époque [VAN 16]. 


\section{Les disparités de la participation dans le niveau d'implication proposé}

Afin d'avoir une idée des actions dans lesquelles sont impliquées les participants au sein de projet de sciences citoyennes, nous nous appuierons sur deux documents qui nous livrent une information commune [BEC 16] ; [WIG 15]. Les auteurs ont analysé la répartition des actions propres à la démarche scientifique proposées aux participants des projets de sciences citoyennes (figure 4, 5).

Il existe une disparité forte entre des actions de collecte d'un côté (collecte de données d'observations principalement) et celles relatives à d'autres étapes de la démarche scientifique notamment l'analyse (analyse et interprétation de données et de graphiques, mais aussi communication de résultats et proposition d'hypothèses) (figure 5). Cette disparité peut être illustrée par le schéma de la PPSR (figure 6) illustrant les 3 étapes de la démarche scientifique dans lesquelles sont impliqués les participants (projets de contribution, projets de collaboration et projet de co-création) avec un degré d'implication de plus en plus fort et une implication complète du citoyen dans les projets de cocreation. Ce sont dans les projets de co-création que les citoyens ont le plus de chance d'exprimer leurs talents, en allant du choix des questions de recherche jusqu'à la discussion des résultats et la définition de nouvelles questions.

Il existe au sein des sciences citoyennes, bien plus de projets correspondant, dans les modèles de PPSR, aux projets de "contribution », essentiellement basés sur la collecte de données que des projets de "co-création" offrant aux citoyens de participer à l'ensemble des étapes du processus de recherche.

Ces résultats participent à la justification du travail mené dans cet article, afin d'identifier les motivations, la diversité et le potentiel de projets d'analyse.

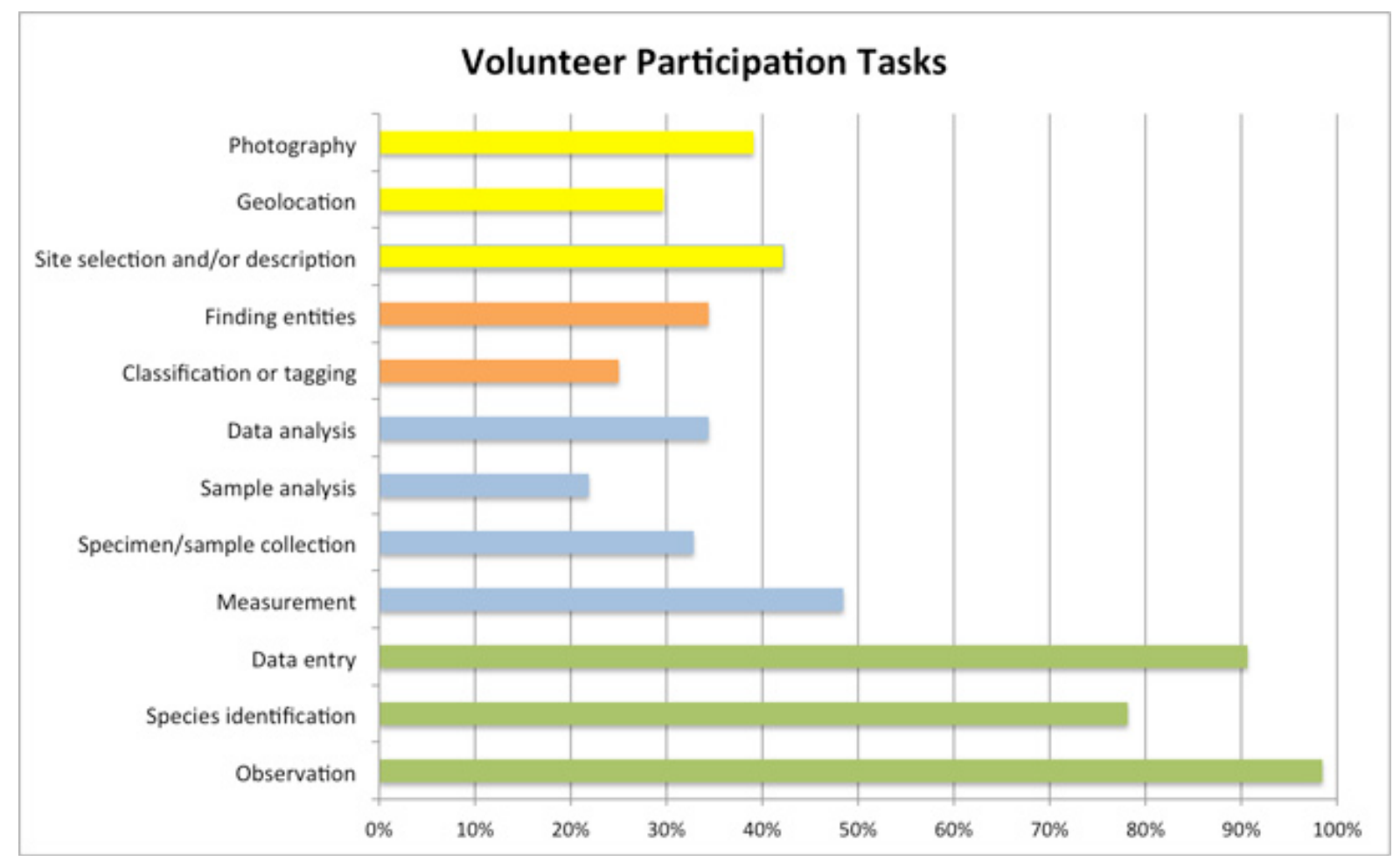

Figure 4. Participation volontaire dans les tâches scientifiques, avec les tâches de collecte de données en vert, les taches de mesures en bleu, les tâches de classification en orange et les tâches d'observation de site en jaune. [WIG 15] 


\begin{tabular}{|c|c|c|c|}
\hline Step in Scientific Process & $\begin{array}{l}\text { Steps included } \\
\text { in Contributory } \\
\text { Projects }\end{array}$ & $\begin{array}{l}\text { Steps included } \\
\text { in Collaborative } \\
\text { Projects }\end{array}$ & $\begin{array}{l}\text { Steps included } \\
\text { in Co-created } \\
\text { Projects }\end{array}$ \\
\hline $\begin{array}{l}\text { Choose or define } \\
\text { question(s) for study }\end{array}$ & & & $\mathrm{x}$ \\
\hline $\begin{array}{l}\text { Gather information and } \\
\text { resources }\end{array}$ & & & $\mathrm{x}$ \\
\hline $\begin{array}{l}\text { Develop explanations } \\
\text { (hypotheses) }\end{array}$ & & & $\mathrm{X}$ \\
\hline $\begin{array}{l}\text { Design data collection } \\
\text { methodologies }\end{array}$ & & $(X)$ & $\mathrm{x}$ \\
\hline $\begin{array}{l}\text { Collect samples and/or } \\
\text { record data }\end{array}$ & $X$ & $\mathrm{x}$ & $\mathrm{x}$ \\
\hline Analyze samples & & $\mathrm{X}$ & $X$ \\
\hline Analyze data & $(\mathrm{X})$ & $\mathrm{x}$ & $x$ \\
\hline $\begin{array}{l}\text { Interpret data and draw } \\
\text { conclusions }\end{array}$ & & $(\mathrm{X})$ & $\mathrm{x}$ \\
\hline $\begin{array}{l}\text { Disseminate conclusions/ } \\
\text { translate results into action }\end{array}$ & $(\mathrm{X})$ & $(\mathrm{X})$ & $\mathrm{x}$ \\
\hline $\begin{array}{l}\text { Discuss results and ask } \\
\text { new questions }\end{array}$ & & & $\mathrm{X}$ \\
\hline
\end{tabular}

$\mathrm{X}=$ public included in step; $(\mathrm{X})$ = public sometimes included in step

Figure 5. Les différents modèles de Participation Publique dans la Recherche Scientifique et leur poids dans l'ensemble des projets de SP. [BON 09] modifié [LHO 16]

\section{Un projet longuement préparé via une série d’ateliers participatifs}

Le projet "analyse citoyenne des données" (ACD) a vu le jour dans le cadre du Groupement de Recherche (GDR) PARCS (Participatory Action Research and Citizen Sciences) ${ }^{6}$ labellisé en 2014 par l'INEE (Institut Ecologie et Environnement) ${ }^{7}$ du CNRS. Il est piloté par un groupe de chercheurs issu de plusieurs laboratoires (CEFE/CNRS, CIRAD, INRIA, INRA) et de représentants d'association naturalistes et/ou coordinateurs de projets de science citoyenne (Tela Botanica, OrchiSauvage, Cybelle Planète, Pl@ntNet [JOL 16]. Il s'est inspiré d'un projet exploratoire nommé "geocollaboratory" qui consistait à connecter à travers toute la planète des initiatives de tourisme autochtone publiées dans un livre [BLA 06] et mises en ligne via un site ${ }^{8}$. A l'aide de cartes interactives, d'un questionnaire co-conçu en ligne et d'un forum de discussion, les communautés hôtes échangent sur leurs bonnes pratiques, ont accès immédiatement aux données générées par le questionnaire en ligne, les enrichissent via le forum et des techniques RAP intégrées sur le site. Le chercheur développe un modèle de recherches participatives à distance entre les communautés [BLA 12]. Ce concept de "web collaboratif" a été retenu comme un des 4 axes prioritaires par le GDR. Il permet de continuer à explorer le potentiel du web pour faire de la recherche à distance entre partenaires éloignés, d'intégrer les outils de la "RAP" en ligne grâce au numérique et de trouver un mode de partenariat chercheur/citoyen moins extractif. Il permet de mettre nos expériences et expertises en commun pour répondre à la question: "Comment impliquer les citoyens dans l'analyse et l'interprétation des données qu'ils contribuent à collecter?» et "Comment mieux répondre aux préoccupations des citoyens pourvoyeurs de données".

\footnotetext{
${ }^{6}$ www.gdrparcs.fr

${ }^{7}$ http://www.cnrs.fr/inee/

${ }^{8}$ Le Guide des Destinations Indigènes. ABORINET: http://aborinet.cefe.cnrs.fr/

(c) 2018 ISTE OpenScience - Published by ISTE Ltd. London, UK - openscience.fr
} 
Le projet a été longuement mûri au sein d'une série de 4 ateliers participatifs menés successivement sur 2 ans. Il a pour but de nous aider à mieux :

- Rééquilibrer les rôles entre scientifiques et non-scientifiques ;

- Etre dans l'action, répondre aux préoccupations citoyennes ;

- Motiver et fidéliser les participants ;

- Intégrer les savoirs des citoyens dans les projets de SP ;

- Apprendre à manipuler des jeux de données scientifiques ;

Trois pistes de réflexion nous permettent d'avancer. Elles sont menées en parallèle (figure 6).

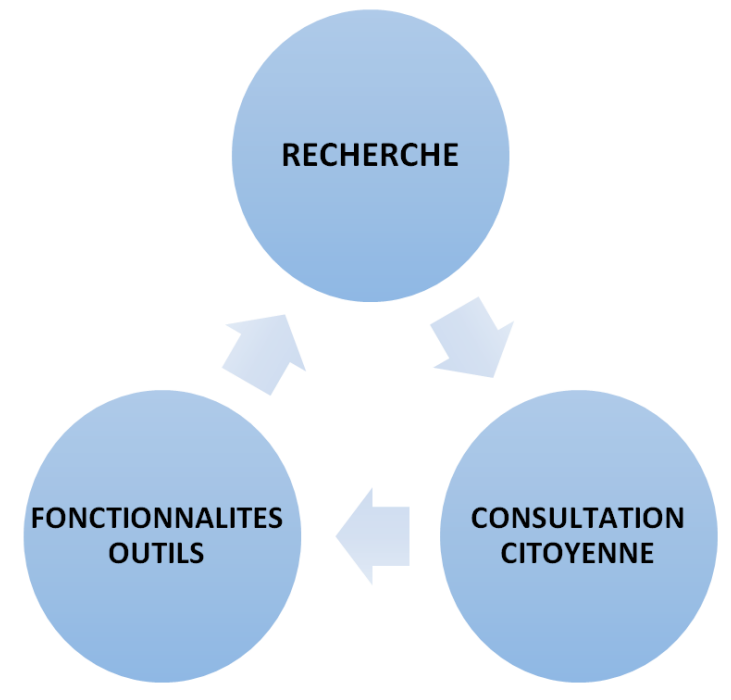

Figure 6. Trois pistes de réflexion interconnectées menées en parallèle pour l'analyse des projets étudiés Voir en annexe 3 les mots-clefs constitutifs de ces groupes. GDR PARCS, 2017.

L'axe « Recherche » vise à questionner les processus et les méthodes développées dans les sites de sciences citoyenne, à analyser les bonnes pratiques et les sites existants. Cela peut se traduire par : Comment les participants s'impliquent et développent un engouement pour les démarches de sciences participatives ? Comment cet engouement perdure dans le temps ? Comment caractériser les relations qui existent entre les différents axes de réflexions (consultation citoyenne, fonctionnalités/outils) au sein des projets?

L'axe « Citoyen » propose d'identifier les besoins et les motivations des participants aux projets de sciences citoyennes et de mesurer leur envie et leur capacité à aller plus loin dans l'analyse.

L'axe « Fonctionnalités et outils » s'intéresse au développement d'outils web et l'intégration de nouvelles fonctionnalités permettant de développer la participation, en amont ou en aval de la fourniture de données, notamment grâce aux outils de la RAP intégrés directement en ligne.

Ce sont les résultats de l'axe "recherche" qui sont présentés ici dans cet article et notamment l'analyse critique des sites de sciences participatives nord-américains identifiés comme source d'inspiration pour les projets des membres du GDR.

\section{La participation au-delà de la collecte des données : caractéristiques et éléments de réussite}

Cette partie est structurée en deux temps : (i) l'étude exploratoire du champ des possibles en termes d'analyse de données et de participation au sein des projets de sciences citoyennes et (ii) la 
caractérisation des conditions de réussite de la participation citoyenne exprimées par des intervenants dans des projets de sciences citoyennes.

\subsection{Approche exploratoire et méthodologie pour l'établissement d'un état des lieux}

Tous les projets de recherche du GDR s'appuient sur les approches de RAP développées par Chevalier et Buckles au Canada [CHE 13] ${ }^{9}$ et sont accompagnés par des sessions de formation annuelle au design et à l'animation d'ateliers. Cette méthodologie repose sur la triple intégration de la recherche, de l'action et de la participation dans toutes les étapes de la démarche scientifique : Conception, Collecte, Analyse, Interprétation, Diffusion, Décision; (CCAIID) (figure 7). Grâce à son solide ancrage théorique, la RAP s'appuie sur une panoplie d'outils permettant de mettre en œuvre, au travers d'ateliers très diversifiés en fonction de la situation que l'on souhaite traiter, une collaboration, une co-construction de la formulation et de la résolution des problèmes identifiés. Ces deux grilles de lecture RAP et CCAIDD nous a permis de mieux analyser les projets retenus.

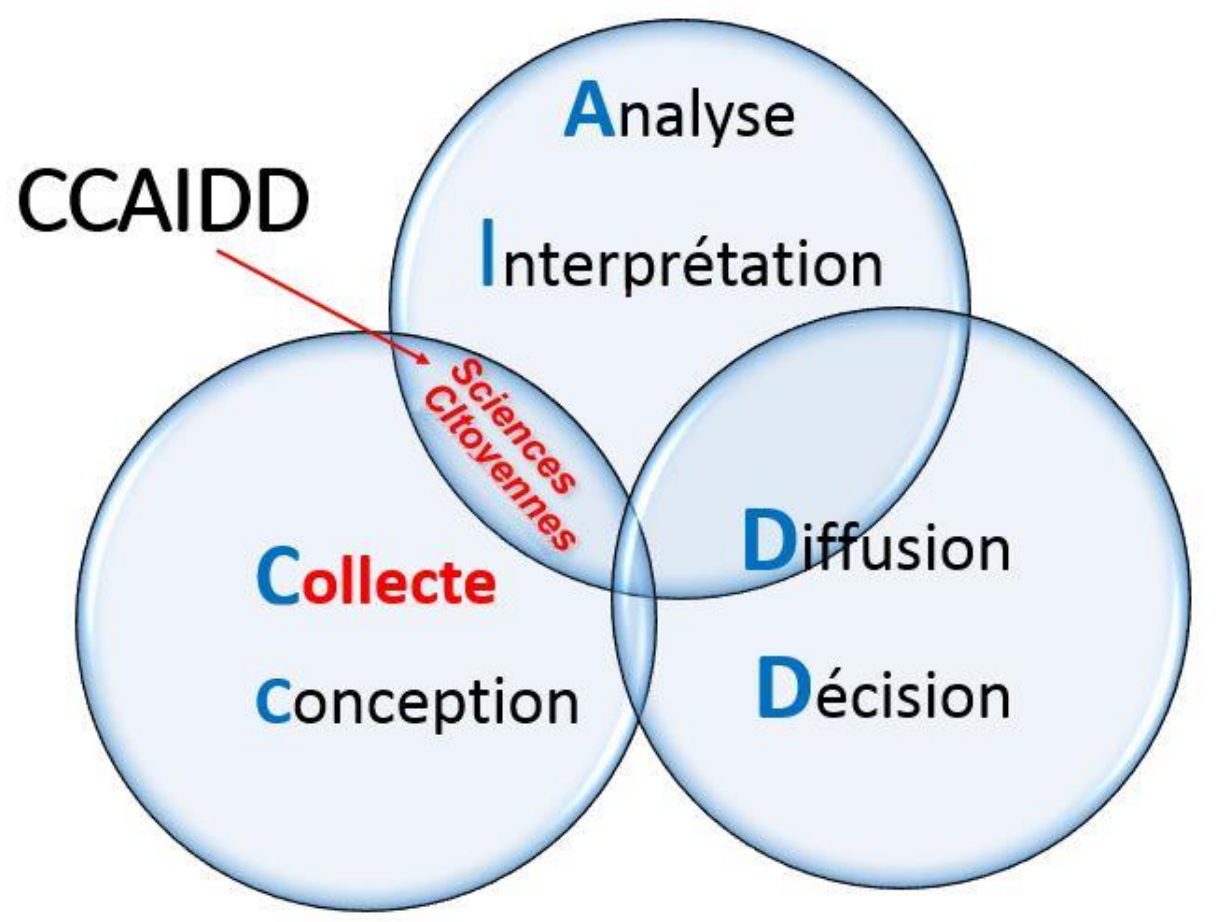

Figure 7. Les 6 étapes d'un projet de recherche action participative: Conception, Collecte, Analyse, Interprétation, Dissémination et Décision (CCAIID). Chevalier, 2013. Modifié, Blangy, 2016.

Nous avons dans un premier temps procédé à un inventaire rapide des projets de sciences participatives en Amérique du Nord. Une grille de critères d'analyse a été développée au fur et à mesure des lectures. Nos critères évolutifs nous ont permis une plus large découverte de ce qui existe et fonctionne au sein de projets de sciences citoyennes. En procédant de cette manière, il a ainsi été possible d'accéder à une représentation fidèle de la diversité des tâches participatives proposées aux citoyens pour l'ensemble des projets étudiés. Nous regroupons nos critères en deux catégories :

- Les critères informatifs de base.

- Les critères relatifs aux actions propres à la participation et qui auront une importance particulière pour notre analyse (tableau 2).

\footnotetext{
${ }^{9}$ https://www.participatoryactionresearch.net/about 
Domaine, discipline (environnement, médecine...)

Objet d'étude (biodiversité, cours d'eau, nuage...)

Pays, région

Nature de l'équipe porteuse (université, association, laboratoire...)

Etat d'avancement du projet et activité (en commencement, peu actif, actif, arrêté)

Implication de la recherche académique (laboratoire et institut de recherche, universités...)

Publications scientifiques (oui/non)

Nombre de contributeurs et observateurs $(0$ $100 ; 100-1000 ; 1000-10000 ; 10000$ -

$100000 ; 100000$ et plus)
Support de la participation, mode de collecte (smartphone, internet, présentiel)

Mode d'Interactions entre les communautés (forum, commentaires, blog, mail chercheurs, ateliers)

Types de données (géolocalisées, visuelles, textuelles, objets graphiques interactifs...)

Mode de visualisation des données (photos, cartes, cartes interactives, graphiques, textes, objets...)

Analyse des données (oui/non)

Objectif de l'analyse (révision, enrichissement, identification, construction, comparaison, validation, planification...) Ludification/jeux (oui/non)

Actions remarquables (création de projet, exportation de données, formation, partage de connaissances scientifiques...)

Tableau 2. Critères de lecture des projets étudiés. [LHO 17]

Deux considérations ont structuré notre approche :

- Chaque projet de SC a été abordé en se mettant dans la situation d'un participant qui découvre le site internet et ce qui lui est possible de faire dans ce projet.

- Il y a « analyse » à partir du moment où le participant peut intervenir et agir (voir tableau 3) sur les données du projet.

Nous avons analysé un total de 31 projets (annexe 4). Les projets ont été étudiés uniquement à travers leurs sites internet, et les publications mises en ligne par les porteurs de ces projets. Les thématiques sur lesquelles portent les différents projets peuvent varier, cependant la majorité d'entre eux sont spécifiques aux domaines de l'environnement. L'environnement intègre tout ce qui peut être relatif à la biodiversité, aux milieux naturels (rivières, parcs...), ou encore à l'aménagement des zones naturelles, etc.

Notre état des lieux et notre analyse porte essentiellement sur des projets hors de France métropolitaine, et sur des zones qui nous sont moins familières dans le domaine des sciences participatives.

\subsection{Caractérisation des différentes formes d'analyse des données}

Les formes d'analyses identifiées dans les 31 projets retenus sont multiples et de plusieurs nature (annexe 5) et combinent les facteurs d'analyses indiqués dans le tableau 3. 


\begin{tabular}{|c|c|}
\hline $\begin{array}{l}\text { Choix de la thématique englobant l'analyse } \\
\text { - Degré de liberté (+) } \\
\text { - Degré de liberté (-) }\end{array}$ & $\begin{array}{l}\text { Encadrement de l’analyse } \\
\text { - Libre } \\
\text { - Accompagnée (tutoriels, ateliers, } \\
\text { protocoles) }\end{array}$ \\
\hline $\begin{array}{l}\text { Origine de la donnée analysée } \\
\text { - Issue des participants } \\
\text { - Issue des porteurs du projet }\end{array}$ & $\begin{array}{l}\text { Interfaces rendant possible l'analyse } \\
\text { - Physique (ateliers en présentiels, } \\
\text { groupes) } \\
\text { - Numérique (statique, dynamique) }\end{array}$ \\
\hline $\begin{array}{l}\text { Objectif de l'analyse } \\
\text { - Caractérisation (identification, révision, } \\
\text { - } \text { spécificité, mesures) } \\
\text { - } \text { pronstruction (géométrique, } \\
\text { - Comparaison/Prévision } \\
\text { - Planification }\end{array}$ & $\begin{array}{l}\text { Interaction entre communautés sur } \\
\text { l'analyse } \\
\text { - Interactions ponctuelles } \\
\text { (commentaires) } \\
\text { - Interactions structurelles (étape du } \\
\text { processus d'analyse) }\end{array}$ \\
\hline
\end{tabular}

Tableau 3. Facteurs conditionnant les formes d'analyse de données. [LHO 17]

\subsubsection{Choix de la thématique englobant l'analyse}

Le degré de liberté laissé au participant dans le choix des thématiques englobant l'analyse peut être fort ou faible. Ce degré de liberté plus ou moins important différencie des projets pour lesquelles la thématique est fixée au départ. Les projets Foldit et Kaggle font partie de ceux dont les thématiques proposées aux participants sont nombreuses. Foldit propose de travailler sur le repliement des protéines, Kaggle de formuler un besoin en terme de programmation sur la reconnaissance d'images, les jeux vidéo ou l'environnement.

D'autres projets sont centrés sur une thématique précise. Les participants peuvent par la suite choisir dans un ensemble de sous-thèmes liés à la thématique principale. Climate Colab traite des enjeux climatiques à l'échelle planétaire. Les participants ont le choix de travailler plus précisément sur la résilience des zones les plus affectées, l'usage des terres, l'approvisionnement énergétique, le prix du carbone ou encore les changements de comportement.

\subsubsection{Origine des données analysées}

Les données analysées peuvent être soit issues des participants soit des porteurs de projet. Certains des projets, le plus souvent ceux dont le degré de liberté dans le choix de la thématique est faible, proposent aux participants de travailler sur des données brutes que les porteurs de projets fournissent. C'est le cas de Snapshot Serengeti, Mozak ou Foldit qui sont des projets portés par des équipes scientifiques sur des thématiques précises (conservation de la biodiversité, système nerveux, protéines) dont l'analyse permet d'alléger grandement le travail de ces derniers. D'autres projets proposent aux participants d'analyser les données collectées par leurs pairs comme dans E-Bird, iNaturalist, NatureNote Book.

\subsubsection{Objectif de l'analyse}

Il s'agit du rôle que va avoir le participant dans l'analyse et sa finalité. Nous en avons identifié quatre : caractérisation, construction, comparaison, planification.

La caractérisation est certainement l'objectif le mieux représenté parmi les projets étudiés. Il s'agit donc de caractériser la donnée brute selon différentes finalités. Cela peut être pour identifier un individu (iNaturalist, Ebird...), renforcer la qualité de l'identification en estimant si elle est juste ou non (iNaturalist, Snapshot Serengeti), évaluer la spécificité d'un évènement climatique s'il est anormal ou non (ISeeChange) ou encore 1'ensemble des caractéristiques via la mesure d'un objet tel que sa taille, son poids, sa couleur, etc., comme pour un lichen par exemple (Particitae). 
La construction vise à produire de nouvelles données, soit à partir de la manipulation graphique de données existantes, soit à partir du développement de nouveaux outils informatiques. Dans le premier cas il s'agit par exemple de la manipulation de protéines dans le projet Foldit pour lui donner la forme convenue, ou encore le traçage des réseaux de neurones dans le projet Mozak. Dans le deuxième cas, la programmation informatique via un jeu de données dans le projet Kaggle permet effectivement de construire un outil informatique répondant aux objectifs fixés par un autre participant pour analyser un jeu de données.

La comparaison est proposée notamment dans le projet Baby Croinc. Il est possible de comparer l'âge de son enfant en lien avec les étapes de son développement (marcher, premiers mots etc.) avec celui d'autres enfants de manière à estimer où se situe son propre enfant au regard de la moyenne. Il est ensuite possible pour les parents constatant un retard d'agir en conséquence et de planifier des activités adaptées au bon développement de l'enfant.

La planification a été identifiée notamment dans le projet Climate Colab. Les participants sont invités à proposer un plan d'action pour répondre à un enjeu relatif au changement climatique. Les participants sont libres de décrire leur projet pour répondre à cet enjeu. Il sera ensuite traité par la communauté des participants selon différentes étapes avant de pouvoir éventuellement être retenu et retravaillé ${ }^{10}$.

\subsubsection{Encadrement de l'analyse}

L'encadrement peut être libre ou encadré. Ce facteur permet d'identifier le niveau d'accompagnement du participant dans l'analyse de données. Un participant peut-être tout seul et libre de mener sa propre analyse, c'est le cas dans l'identification d'une espèce ou dans la caractérisation d'un évènement climatique. A l'inverse, le participant peut aussi être accompagné de façon plus ou moins importante via des tutoriels (Snapshot Serengeti), des protocoles de mesures (Teatime 4 Science) ou encore des ateliers en présentiel avec un accompagnement scientifique dans l'analyse (Survivor).

\subsubsection{Interfaces à travers laquelle s'effectue l'analyse}

Les interfaces rendant possible l'analyse peuvent être physiques (ateliers en présentiel) ou numériques (ateliers en virtuel via un site internet). Pour l'interface physique le participant a physiquement accès à la donnée. Il peut s'agir aussi bien de mesures pour caractériser un sujet que de réunions en présentiel au cours desquelles les participants regroupés ensemble travaillent sur l'analyse des données qu'ils ont collectées en amont. L'interface numérique passe par l'utilisation d'une plateforme internet ou d'une application smartphone pour réaliser l'analyse de données. Nous différencions les interfaces numériques dites «statiques» de celles dites «dynamiques» en fonction du niveau de complexité de l'interface en question. Une interface classique avec des boutons, onglet et barres de textes sera considérée comme statique (Snapshot Serengeti, iNaturalist, Cochrane Crowd...) et une interface basée sur la représentation graphique d'une forme que l'on peut manipuler dans l'espace ou qui permet la consultation d'animations visuelle sera plutôt considérée comme dynamique (Foldit, Mozak, Stallcatcher).

Il existe à la fois des projets uniquement basés sur une interface numérique (Snapshot Serengeti, Amazon Aerobotany, Mozak...), ou uniquement basés sur une interface physique (Survivor) et des projets combinant les deux interfaces (Nature Note Book, Shale Gas Monitoring project, Georgia Adopt-A-Stream...).

\footnotetext{
${ }^{10}$ https://www.climatecolab.org/ 
Il peut y avoir plusieurs types d'interaction entre communautés que ce soit entre participants seulement ou entre participants et scientifiques. Les interactions ponctuelles peuvent avoir lieu à la suite de l'analyse pour corriger ou échanger à propos de cette dernière, via la possibilité de faire des commentaires par exemple. Les interactions structurelles conditionnent le processus d'analyse, c'est-àdire que le retour et l'évaluation des autres participants est une étape nécessaire indispensable à la progression de l'analyse (Climate Colab).

\subsection{Les actions remarquables favorisant la participation et l'engagement et allant au-delà de l'analyse}

Nous avons dans un premier temps centré nos recherches sur les différentes formes que peuvent prendre l'analyse des données. Au cours de nos consultations des sites de Sciences citoyennes, nous avons très vite identifié d'autres types d'intervention qui favorisent et pérennisent la participation des citoyens. Ces actions et dispositifs proposés aux citoyens nous semblent être remarquables du point de vue de la participation sans être de l'analyse à proprement parler. Elles sont classées selon trois axes principaux synthétisés dans le tableau 4. (La montée en compétence, l'autonomisation et l'engagement).

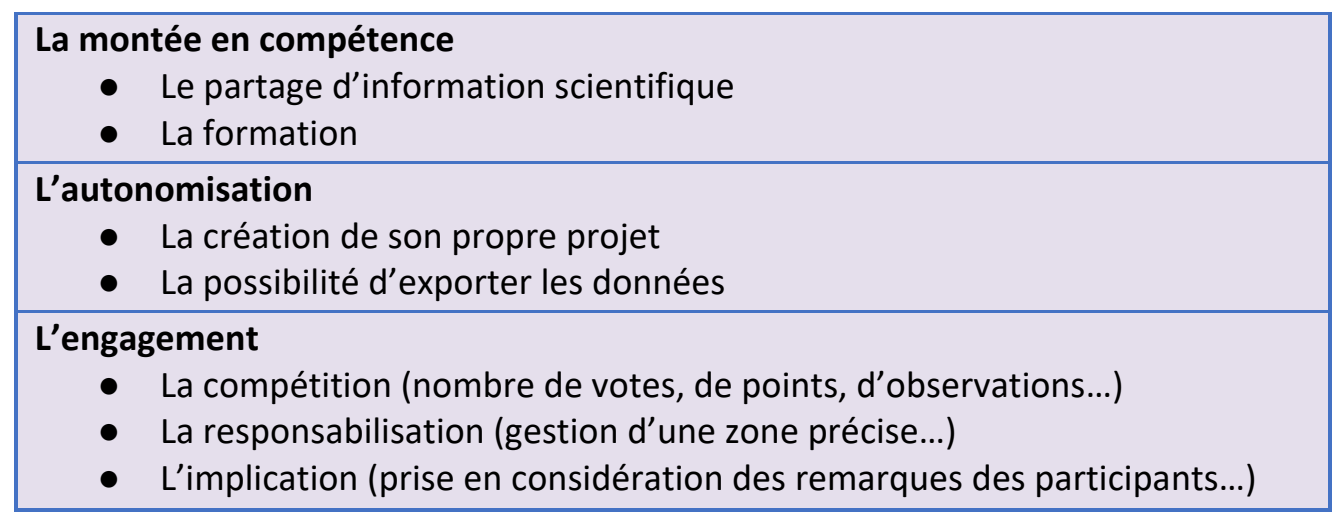

Tableau 4. Trois thèmes permettant de mieux caractériser les actions remarquables qui favorisent la participation. [LHO 17]

Le premier axe concerne la montée en compétence. De nombreux projets proposent en échange des données fournies, de partager des informations scientifiques permettant aux citoyens impliqués de se familiariser plus en profondeur avec la thématique (ISeeChange). D'autres projets proposent aussi des formations accessibles en ligne ou en présentiel (Nature Note Book, GROW).

L'autonomisation est une dimension importante de la participation car elle permet aux participants de s'approprier un sujet scientifique et de le traiter. Ainsi, l'action la plus remarquable est caractérisée par la possibilité de créer son propre projet. Les participants définissent ainsi leur protocole, les objets qu'ils veulent traiter, etc. Une autre action qui va dans le sens de l'autonomisation est la possibilité d'exporter les données. Les participants ont alors la possibilité d'effectuer leur propre analyse hors des outils proposés par le projet.

Enfin, le dernier élément qui intervient de façon importante dans la participation est la notion d'engagement. Il s'agit des moyens qui vont être mis en place pour favoriser l'engagement des participants dans les projets de science citoyenne de façon la plus durable et pérenne possible. Rappelons que les gestionnaires de site de sciences citoyennes souhaitent fidéliser leurs pourvoyeurs de données. La manière la plus courante de fidéliser les participants est la compétition. Que ce soit par le vote, le nombre de points, le nombre d'observations, l'objectif est d'améliorer son score par rapport aux autres participants. La compétition va stimuler l'engagement dans le projet. D'autres vecteurs d'engagement existent comme la responsabilisation dans le projet The Shale Gas Monitoring Project 
ou Georgia Adopt-A-Stream, les participants se voient accorder un tronçon de rivière et deviennent responsables de la caractérisation de ces tronçons via plusieurs types de mesures. Chaque participant est responsable d'une petite partie d'un projet bien plus large. Enfin le degré d'implication reste très difficile à mesurer. Il n'est pas visible ni mis en exergue sur les sites internet analysés. Il s'agit d'intégrer certaines remarques ou questionnement des participants dans la réflexion scientifique par exemple dans la formulation de la question de recherche. C'est ce qui est le cas dans le projet Shale Gas Monitoring Project.

\subsection{Propositions pour une typologie de projets}

Sur la base de l'analyse des 31 projets distincts, nous proposons une typologie des projets qui nous permette de mesurer et caractériser la participation aux différentes étapes de la démarche de recherche action participative (voir le CCAID). Nous avons donné un nom à chacune des familles de projets qui tient compte du niveau de participation des participants : holistique, structurée, limitée et extractive.

Nous illustrerons chaque type de participation par la description succincte d'une initiative retenue dans notre étude.

\subsubsection{Participation holistique - The Shale Gas Monitoring Project}

Un article récent [WIL 16] décrit de façon très détaillée les étapes de développement antérieures au projet "Shale Gas Monitoring Project" Nous qualifions la participation des non-scientifiques à ce type de projet de «holistique » dans la mesure où elle est véritablement intégrée dans le système de réflexion scientifique développé par l'association qui porte le projet.

L'association ALLARM (Alliance for Aquatic Resource Monitoring) a développé une méthode de participation qui nous semble être l'une des plus avancée parmi celles que nous avons pu analyser. Elle offre l'opportunité aux citoyens de s'impliquer dans le plus d'étapes du CCAID.

Le projet «Shale Gas Monitoring Project» consiste à évaluer l'impact des puits de gaz informels sur les cours d'eau d'une région des Etats-Unis. L'orientation qu'a prise le projet vient des observations réalisées par les participants impliqués dans les projets précédents. La question à l'origine du projet a donc été co-construite par les participants précédemment impliqués, les scientifiques et les étudiants intervenants au sein de l'association qui ont organisé par la suite différents ateliers (61 ateliers au total) en présentiel de façon à construire avec les citoyens la méthodologie qu'ils souhaitent suivre pour réaliser ce travail. Plusieurs étapes sont proposées selon un cycle illustré dans la figure 8 .

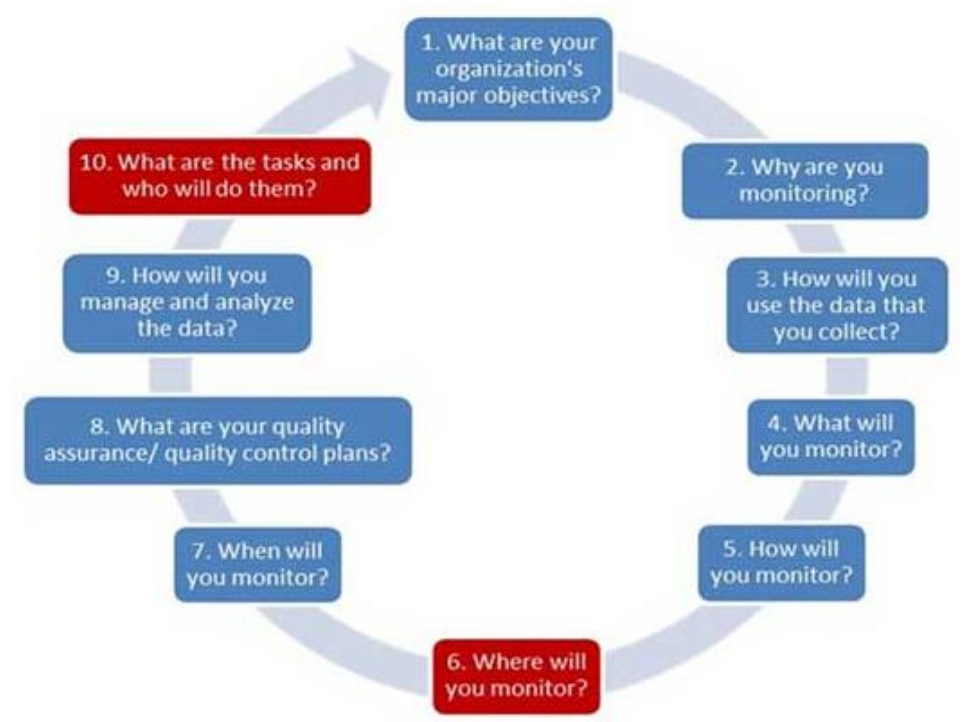

Figure 8. Cycle méthodologique suivi par l'association ALLARM dans ses projets. http://blogs.dickinson.edu/marcellusmonitoring/files/2012/08/Shale-Gas-Volunteer-Monitoring-Manual-2.0.pdf 
L'association mets en ligne à disposition des participants, des outils ainsi que des documents d'accompagnement dans les mesures à effectuer. D'autres ateliers sont organisés pour restituer les mesures de chacun, les analyser ainsi que les interpréter. Les manuels mis à disposition des participants expliquent via un arbre à décision ce qu'il est recommandé de faire dans certaines situations. Les observations effectuées par les participants sont fiabilisées grâce à une étape de vérification qualitative des manipulations en comparant un échantillon caractérisé par le participant et le même échantillon caractérisé par un laboratoire (figure 9).

Les participants sont ainsi impliqués dans la totalité des étapes de la démarche scientifique, de la conception de la question à la diffusion des résultats voir même aux décisions politiques qui peuvent être prises par la suite.

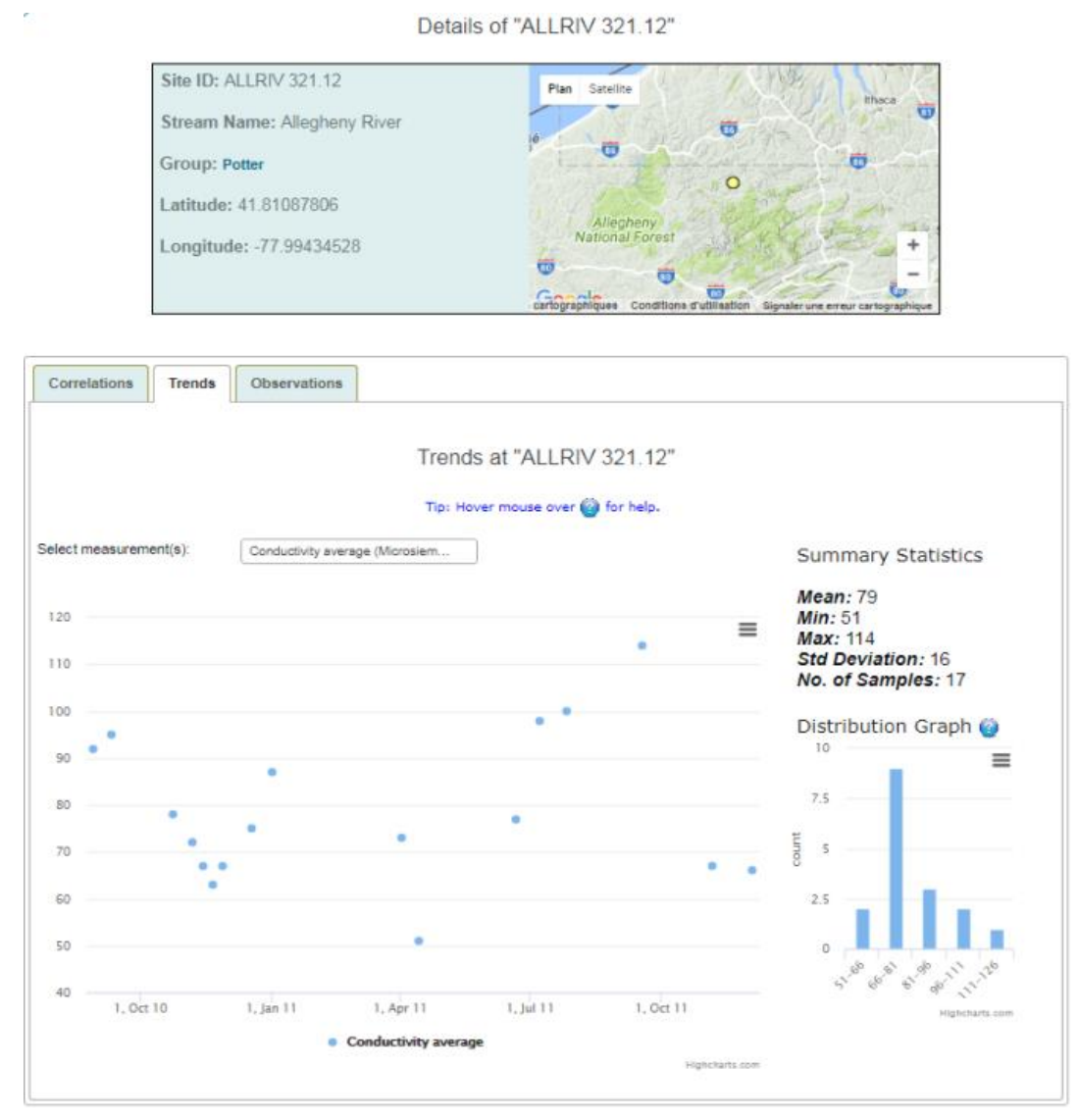

Figure 9. Interface numérique du projet Shale Gas Monitoring Project http://allarmwater.org/cwis438/websites/allarm/RegionData.php?WebSitelD=12 - 06.2017

\subsubsection{Participation structurée - Nature Note Book}

Dans le registre de l'identification de caractéristiques phénologiques du vivant, le projet Nature Note Book propose une approche différente du projet. Il se présente comme une application mobile couplée à un site internet qui référence l'ensemble des observations photographiques réalisées par les participants. Classiquement, les participants peuvent proposer une identification de l'espèce observée ou réviser les identifications des autres participants. Ce projet offre de plus la possibilité aux participants de monter des groupes et d'organiser des «projets locaux». Les groupes ainsi constitués peuvent se voir accorder un filtre à leur nom de groupe dans les outils de visualisation des données du site internet (cartes, graphiques, ...). De plus, de nombreuses ressources sont disponibles sur le site internet pour permettre aux différents groupes, qui peuvent être des associations écologistes, des écologistes amateurs ou même des classes, d'organiser des ateliers en présentiel.

Ces ressources peuvent être des PowerPoint dans lesquels le contenu est adapté à différents usages comme l'introduction à la phénologie par exemple. Le projet Nature Note Book met donc en œuvre 
beaucoup de moyens pour permettre aux participants de s'approprier les outils (application, visualisation des données, informations académiques...) dans des référentiels locaux, solliciter l'organisation en groupe et l'auto-formation entre les participants. De fait la participation est clairement structurée par le projet dans la mesure où il y a une volonté d'impliquer les participants, de leur permettre de développer leurs projets, de monter en compétence et d'être autonome dans l'utilisation qui peut être faite des outils. Pour autant, la participation se différencie du projet précédent car les réflexions scientifiques menées en arrière-plan par les équipes porteuses du projet à savoir l'USA National Phenology Center, sont indépendantes des activités menées par les participants. Ces dernières sont structurées par le projet mais ne sont pas partie intégrante du système de réflexion scientifique à l'origine du projet.

\subsubsection{Participation limitée - Tea Time 4 Science}

Pour d'autres types de projet, la participation est limitée à une seule étape bien précise du projet scientifique. Souvent, dans ce type de projet, les participants sont conscients qu'ils n'interviennent qu'à un moment bien précis du projet en appliquant un protocole fourni par les porteurs de projet. La participation est donc clairement limitée à une action qui ne pourrait être réalisée d'une autre manière que par l'implication des participants non-scientifiques. Beaucoup de projets pourraient illustrer ce type de participation. Le projet Tea Time 4 Science invite les participants à enterrer des sacs de thé autour de chez eux afin d'évaluer la dynamique de décomposition de la matière organique dans les sols. Par la mobilisation de nombreux participants à travers le monde, les scientifiques peuvent décliner leurs recherches avec beaucoup plus de paramètres en fonction des pays, des sols et des conditions climatiques in situ. Dans ce projet, les scientifiques ont mis à disposition des participants un protocole relativement précis qui décrit les conditions dans lesquelles doivent être enterrés les sacs de thés, comment les peser, les sécher au four, etc. En dehors de ces mesures analytiques, les participants ne sont pas consultés. Leur participation est limitée à cette étape de la démarche scientifique. Les participants reportent ensuite les mesures qu'ils ont faites sur le site internet du projet qui centralise les mesures effectuées par l'ensemble des participants.

\subsubsection{Participation extractive - Snapshot Serengeti}

Nous qualifions les projets dont la participation nous semble être la moins développée de participation extractive. Dans le projet Snapshot Serengeti. Les participants accèdent à un portail internet sur lequel a été développée une interface très ergonomique et fonctionnelle, illustrée par la figure 10.

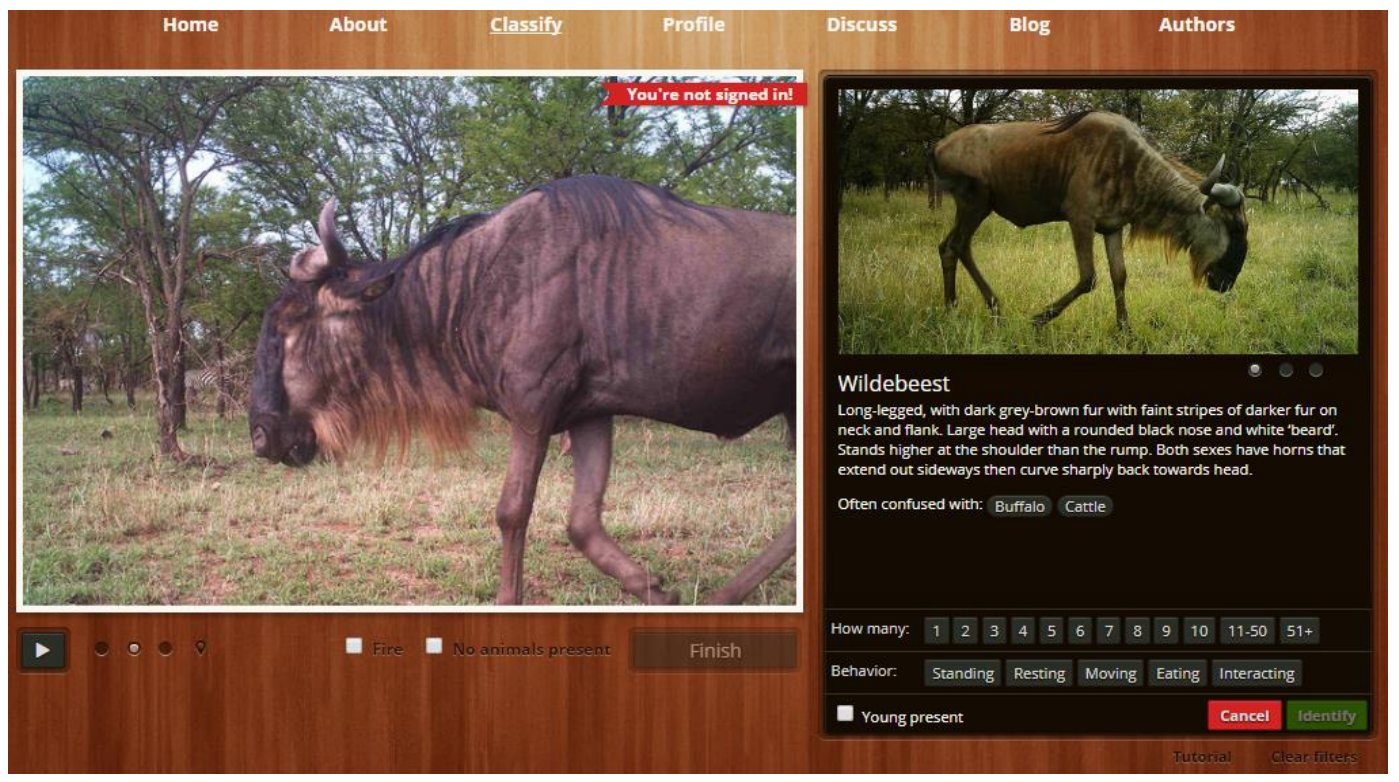

Figure 10. Interface numérique proposée par le projet Snapshot Serengeti. https://www.snapshotserengeti.org/ - 06.2017 
Accompagné par un tutoriel, les participants procèdent à un étiquetage par étape pour identifier les espèces présentes sur des photos. La donnée analysée n'est pas collectée par les participants mais fournie par les porteurs de projet (à travers des pièges photographiques). Les données viennent d'un parc naturel en Tanzanie qui travaille en collaboration avec l'Université du Minnesota aux Etats-Unis. L'objectif est d'avoir un suivi des flux des espèces animales du parc pour optimiser leur conservation. Nous considérons donc la participation comme étant extractive dans la mesure où le participant n'a besoin de rien d'autre que d'une connexion internet, de suivre un tutoriel très court et très clair avant de répéter à la chaîne les actions d'interprétation photographique. Quelques informations sont diffusées sur le blog du projet pour connaitre l'usage qui est fait des analyses réalisées ou pour donner quelques conseils d'identification.

\subsubsection{Répartition des 31 projets dans les quatre types}

Une rapide classification sur la base des informations accessibles sur les sites web (annexe 4) des 31 projets donne : 2 projets à participation holistique, 8 projets à participation structurée, 10 projets à participation limitée et enfin 9 projets à participation extractive. Deux projets n'ont pas pu être classés, le projet DataOne pour lequel cette typologie n'avait pas grand sens, et un second site pour lequel il manquait les informations qui auraient permis de le classer. Bien que cet échantillon ne soit sans doute pas représentatif de tout ce qui se fait actuellement, et qu'une analyse statistique soit discutable de ce fait, il ressort cependant de cette première typologie sur 31 projets que les projets à participation holistique sont encore rares, et qu'une majorité de projets sont encore caractérisés par des participations extractives ou limitées. Un autre constat est le décalage pour quelques projets entre un discours déclaratif qui pourrait laisser penser à une participation holistique sur la page d'accueil du site web et la nature limitée ou extractive de la participation demandée dès que l'on s'inscrit pour participer à des actions précises de science citoyenne.

\section{Une complémentarité entre espace numérique et présentiel pour une participation citoyenne plus engagée}

La participation des citoyens au sein des projets de sciences citoyennes est caractérisée par l'implication de ces derniers dans les différentes étapes de la démarche scientifique (figure 11). Il existe des projets pour lesquels la participation citoyenne est la plus «développée ». Elle consiste à participer à toutes les étapes de la démarche scientifique. Cela se matérialise par la mise en œuvre simultanée de certaines formes d'analyse et d'actions remarquables comme décrites précédemment. Il est donc possible de définir une typologie des projets en fonction du niveau de participation qu'ils proposent (voir annexe 4).

Dans les projets dont le niveau de participation est le plus élevé, une complémentarité entre une interface numérique et une interface physique est offerte. C'est le cas pour les projets caractérisés par une participation « holistique » et «structurée » (annexe 5). Dans ces projets, l'organisation d'atelier en présentiel permet soit de définir les orientations de la réflexion scientifique du projet (The Shale Gas Monitoring Project), soit de favoriser l'autonomie et l'usage des outils développés par le projet (Nature Note Book). L'interface numérique quant à elle, permet de centraliser les données et les participants sur une plateforme commune à grande échelle. Muki Haklay [HAK 16] du groupe de recherche «Extreme Citizen Sciences» $(\text { ExCiteS) })^{11}$, dans sa présentation sur les «Sciences Citoyennes Participatives », propose une représentation pyramidale de la participation dans les projets de sciences citoyennes (figure 10).

\footnotetext{
${ }^{11}$ https://uclexcites.wordpress.com 


\section{Participation in Citizen Science}

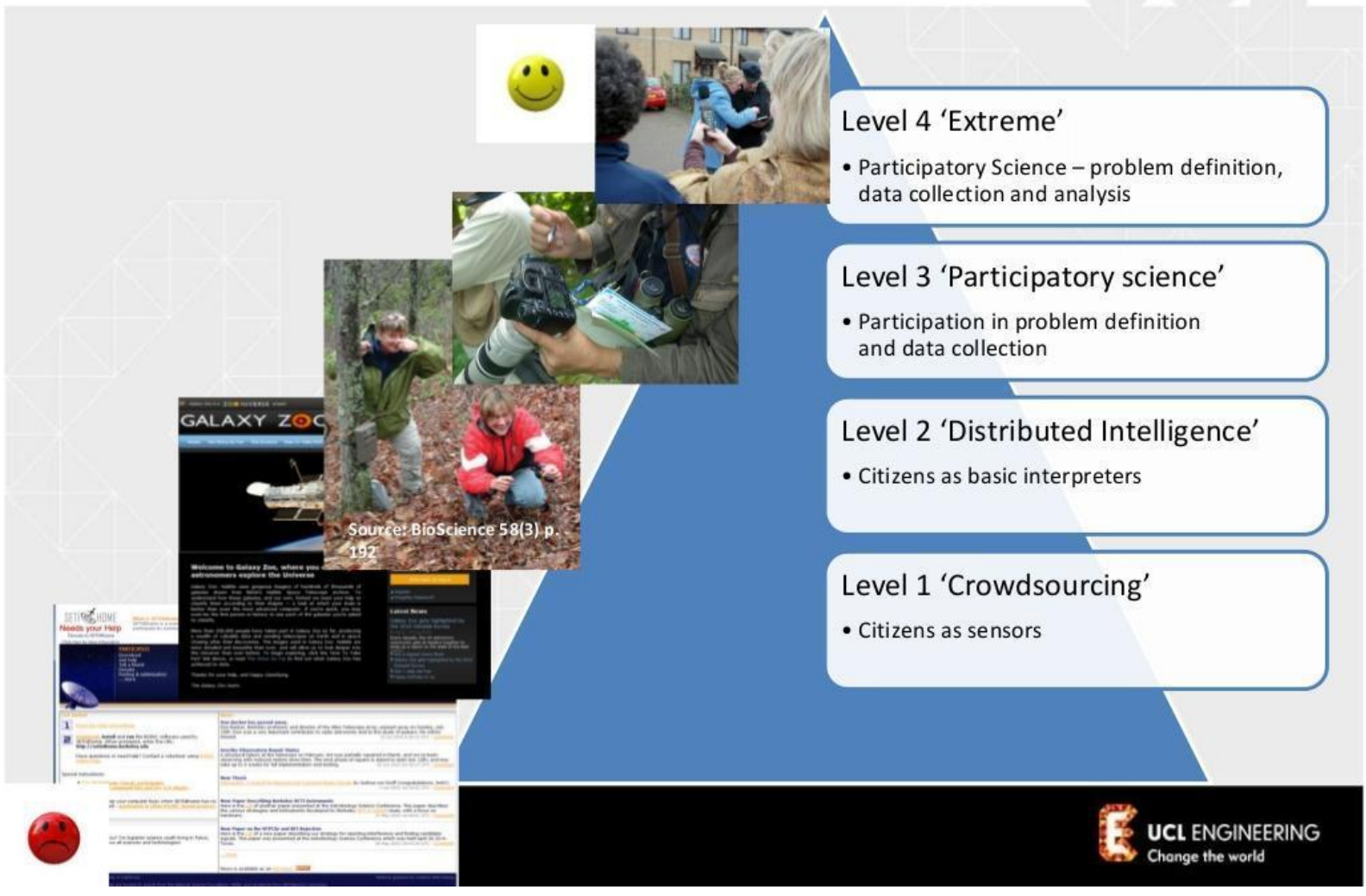

Figure 11. Représentation pyramidale du niveau de la participation dans les sciences citoyennes [HAK 16]

A la base de la pyramide, là où le niveau de participation est le plus faible, l'image de référence est une interface numérique. Là où il est le plus élevé, les images de référence illustrent des activités en présentiel (interface physique).

Un projet qui se veut très participatif à chaque étape de la démarche scientifique ne doit pas impérativement rassembler beaucoup de participants. A l'opposé, des projets dont le niveau de participation est faible mais qui regroupent énormément de participants ont recours à d'autres vecteurs d'engagement que celui de la participation.

Les sciences citoyennes ont initialement permis aux scientifiques de mener des recherches à plus grande échelle et dans un laps de temps beaucoup plus court en proposant une participation citoyenne centrée sur la collecte de données. Les participants pourvoyeurs de données ont permis aux scientifiques d'aller plus vite, d'être plus souvent présents en tant qu'observateurs sur le terrain, de couvrir des territoires d'étude plus étendus. Cette première forme de participation a eu et continue d'avoir des impacts scientifiques et économiques majeurs. Le développement de la participation et de l'implication des citoyens à toutes les étapes de la démarche scientifique, permet de développer et de prendre en considération les enjeux éducatifs ou sociétaux. La participation au-delà de la collecte de données du citoyen ne permet pas seulement de gagner du temps, mais de monter aussi en compétence, de s'approprier la démarche scientifique, d'agir et de prendre part aux thématiques de recherche, thématiques dans lesquelles leur participation était faible jusqu'à présent.

\section{Conclusions}

Nous pouvons retenir trois pistes principales qui aident à mieux connaitre les projets de science citoyenne et l'implication des participants à l'analyse de données. 
La première consiste à trouver un temps de présence entre les participants afin de pouvoir directement échanger, discuter et formuler ensemble la question de recherche. Ce moment de rencontre n'est pas toujours facile à organiser quand les participants sont éloignés les uns des autres. Des rencontres en présentiel sous une forme participative permettent une articulation efficace avec les outils numériques mis à disposition.

Une deuxième piste concerne la co-construction, de bout en bout, du projet de recherche. La volonté de travailler de façon collaborative et horizontale entre participants et chercheurs, où chacun exprime ses idées et où tout le monde décide des règles à observer, semble également un facteur de réussite très important pour l'appropriation et la fidélisation des participants à un projet.

Enfin, la dernière piste concerne les différentes formes d'utilisation de supports numériques pour faciliter la montée en compétence et impulser la mobilisation en présentiel de sorte à ce que le numérique et le présentiel deviennent les plus complémentaires possibles. Le numérique devient ainsi un outil à la base d'une organisation entre participants sur une problématique identifiée par ces derniers. Il peut permettre à des non-experts de s'approprier le fruit de méthodes scientifiques complexes via des considérations ergonomiques et pédagogiques intégrées. Le développement d'outils numériques, à la fois performants et largement accessibles, nécessitent des expertises et des moyens financiers conséquents. Il convient donc de penser leur développement de manière la plus générique et modulaire possible, afin d'en permettre une large réutilisation par différents programmes.

Les sciences citoyennes connaissent un développement majeur en France et dans de nombreux autres pays comme les Etats-Unis, le Canada, l'Australie ou encore l'Angleterre [STO 12] [HOU 16]. L'intérêt que suscite cette forme de démarche scientifique à la fois chez les chercheurs et les citoyens participants génère des questions notamment dans la compatibilité de ces intérêts. Certains projets se veulent extractifs, le revendiquent et proposent des interfaces ludiques, ergonomiques et instructives aux participants tout en leur permettant de faire avancer la recherche notamment dans le domaine médical. Mais la participation peut aussi être plus intégrale au niveau de l'ensemble des étapes de la démarche scientifique plutôt qu'à l'unique étape de collecte de données. Nous avons choisi de centrer nos investigations sur les projets cherchant à développer une démarche participative plus intégrale en identifiant les moyens qu'ils mettent en place pour permettre aux participants d'accéder à l'analyse des données mais aussi à leur interprétation, à la formulation d'hypothèses etc. La collecte de donnée par les usagers ou individus, amateurs ou non, n'est pas nouvelle, mais renouvelée par les technologies de l'information ou cognitives en réseau. Le «tournant participatif » est lié à une mutation plus profonde de la philosophie politique d'une part et d'autre part d'évolutions dans les dynamiques de pouvoir des démocraties de type occidental [THO 08].

Suite à l'état des lieux que nous avons mené sur les 31 projets de science citoyenne, il apparait qu'une corrélation existe entre une participation élevée (à chaque étape de la démarche scientifique) et l'utilisation complémentaire d'une interface numérique et physique en présentiel. L'espace numérique permet d'étendre des projets à plus grande échelle tout en facilitant la mobilisation et l'organisation des participants. L'espace présentiel permet d'assurer un ancrage local, c'est-à-dire l'association du projet à un cadre de référence voir à une problématique identifiée dans l'environnement dans lequel évoluent les participants. De fait, cela permet aux projets d'avoir une plus grande résonnance dans les projets individuels ou collectifs des participants. Enfin, le présentiel a l'avantage de permettre un accompagnement, un suivi et une montée en compétence plus rapide et efficace qu'un accompagnement en ligne via un espace numérique. L'utilisation simultanée de ces deux espaces permet aux projets d'atteindre un degré de participation élevé aussi bien au niveau quantitatif que qualitatif.

Ces pistes identifiées sont importantes pour la mise en œuvre de projets de science citoyenne voulant se constituer sur une base participative solide. Ces pistes vont nous servir dans la phase 2 (consultation citoyenne) et 3 (outils et fonctionnalités) de notre programme Analyse Citoyenne des 
Données. (figure 6) et nous permettrons de continuer à progresser pour mieux intégrer les citoyens dans nos projets. Pour autant, d'autres axes de réflexions restent à éclaircir pour optimiser cette mise en œuvre. Le premier et certainement le plus important est l'évaluation des besoins exprimés par les participants aux projets de science citoyenne eux-mêmes.

\section{Remerciements}

Nous remercions le labex CEMEB et le labex DRIIHM pour le soutien financier à ce projet ACD, pour le financement des formations à la Recherche Action Participative et l'INEE du CNRS pour la labélisation du GDR PARCS. Le travail de Valentin Lhoste a été réalisé dans le cadre du projet Floris'Tic (PIA 2015 - 2018 soutenu par l'ANRU et coordonné par Agropolis Fondation).

\section{Bibliographie}

[BLA 12] BLANGY S., HOLLY M. D., SCOTT M., « Developing a geocollaboratory for Indigenous tourism research », Current Issues in Tourism, v. 15, $\mathrm{n}^{\circ}$ 7, p. 693-706, 2012

[BLA 06] BLANGY S. Le Guide Des Destinations Indigènes, INDIGENE EDITIONS, Collection Indigène Esprit. 384 pp.

[BEC 16] BECKER-KLEIN et al. Embedded Assessment as an Essential Method for Understanding Public Engagement in Citizen Science. Citizen Science: Theory and Practice, 1(1): 8, pp. 1-6, 2016. DOI: http://dx.doi.org/10.5334/cstp.15

[BON 96] BONNEY R., « Citizen Science: a lab tradition », Living Bird, v. 15, n 4, p. 7-15, 1996.

[BON 09a] BONNEY R., BALLARD H., JORDAN R., Mc CALLIE E., PHILIPPS T., SHIRK J., WILDERMAN C., «Public Participation in Scientific Research: Defining the field and assessing its potential for informal science education. A CAISE Inquiry Group Report. », Washington D.C.: Center for Advancement of Informal Science Education (CAISE), 2009.

[BON 09b] BONNEY R., COOPER B. C., DICKINSON J., KELLING S., PHILlIPS T., ROSENBERG V. K., SHIRK J., «Citizen science: a developing tool for expanding science knowledge and scientific literacy », BioScience, v. 59, $\mathrm{n}^{\circ}$ 11, p. 977-984, 2009.

[CHE 13] CHEVALIER J. M., BUCKLES D., Participatory action research: Theory and methods for engaged inquiry, Routledge Editions, Londres, 2013.

[CSA 2017a]. Citizen Science Alliance. 2017. «Citizen Science; added to the Oxford English Dictionary ». Consulté le 11 juillet 2016. http://greatbarrierreefcitizenscience.org.au/news/citizen-science-added-to-the-oxford-englishdictionary.

[CSA 2017b] «About - CitizenScience.orgCitizen Science». CitizenScience.org. Consulté le juillet 11. http://citizenscience.org/about/.

[BEC 16] Becker-Klein R., PETERMAN K., STYLINSKI C., « Embedded assessment as an essential method for understanding public engagement in citizen science », Citizen Science: Theory and Practice, v. 1, n1, p. 8, 2016.

[GAN 17] GANZEVOORT W., VAN DEN BORN R.J.G., HALFMANN W., TURNHOUT S., « Sharing biodiversity data: citizen scientists' concerns and motivations », Biodiversity and Conservation, n 26, p. 2821-2837, 2017.

[HOU 16] HOULLIER F., MERILHOU-GOUDARD J. B., Les sciences participatives en France: Etats des lieux, bonnes pratiques et recommandations, Les sciences participatives en France, 2016.

[IRW 95] IRWIN A. Citizen science: A study of people, expertise and sustainable development, Routledge Editions, Londres, 1995.

[JOL 16] JOLY, A., BONNET P., GOËAU H., BARBE, J., SELMI S., CHAMP J., DUFOUR-KOWALSI S., AFFOUARD A., CARRE J., MOLINO J. F., BARTHELEMY D., BOUJEMAA N., « A look inside the Pl@ ntNet experience». Multimedia Systems, v. 22, nº6, p. 751-766, 2016.

[CHE 09] CHEVAliER, J., BUCKLES, D., BOURASSA, M., «Guide de la recherche-action, la planification et l'évaluation participatives ». ,2009. 
[CHE 09] CHEVALIER, J., BUCKLES, D., BOURASSA, M., «Guide de la recherche-action, la planification et l'évaluation participatives ». ,2013

[LHO 17] LHOSTE, V.. Ouvrir le champ de l'analyse des données via la participation. Exploration des possibilités fournies par les projets de science citoyenne. Rapport réalisé dans le cadre du certificat d'étude tutoré de Montpellier Sup'Agro. Tuteur de stage : Sylvie Blangy (CEFE/CNRS) et Pierre Bonnet (AMAP/CIRAD). 2017.

[MER 16] MERCIER E., DUSSEAUX V., Les Français et les sciences participatives, Une enquête Ipsos Sopra Steria, Préparé pour La Recherche et Le Monde, IPSOS, 2016.

[HAK 16] HAKLAY M., «Participatory [Citizen] Science», First International ECSA Conference, Berlin, 2016.

[HAK 14] HAKLAY M., SHAM P.V., Citizen Science in Oxford English Dictionary, personal blog, 2014.

[KUL 16] KULLENBERG C., KASPEROWSKI D. "What is citizen science?-A scientometric meta-analysis". PloS one, 11(1), 2016.

[OED 17] OXFORD ENGLISH DICTIONARIES. « citizen science - definition of citizen science in English | Oxford Dictionaries ». Consulté le 11 juillet 2017. https://en.oxforddictionaries.com/definition/citizen_science.

[ROW 05] ROWE G., FREWER L. J.,« A typology of public engagement mechanisms », Science, Technology, \& Human Values, v. 30, $\mathrm{n}^{\circ}$ 2, p. 251-290, 2005.

[SER 16] SERVAJEAN M., JOLY A., SHASHA D., CHAMP J., PACITTI E., « ThePlantGame: Actively Training Human Annotators for Domain-specific Crowdsourcing », Dans Proceedings of the 2016 ACM on Multimedia Conference, p. 720-721, Amsterdam, 2016.

[THO 08] THORPE C. « Political theory inSciences and technology studies, in E.J.Hacket \& Alii, The Handbook of science and technology studies », MIT Press, 2008. third edition pp 63-83

[SHI 12] SHIRK J., BALLARD H., WILDERMAN C., PHILIPS T., WIGGINS A., JORDAN R., McCALLIE E., «Public Participation in Scientific Research: A Framework for Deliberate Design ». Ecology and Society, v. 17, n 2, 2012.

[STO 12] STORUP B., MILLOT G., NEUBAUER C., La recherche participative comme mode de production de savoirs. Un état des lieux des pratiques en France, 2012.

[VAN 16] VAN DER LEEUW S., La science, les politiques et le public : quelle réalité, quels écueils ? », Natures Sciences Sociétés 2016/2 (Vol. 24), p. 160-167.DOI 10.1051/nss/2016014

[WIG 15] WIGGINS A., CROWSTON K., « Surveying the Citizen Science Landscape ». First Monday, v. 20, n ${ }^{\circ}, 2015$.

[WIL 16] WILDERMAN C., MONISMITH J., « Monitoring Marcellus: A Case Study of a Collaborative Volunteer Monitoring Project to Document the Impact of Unconventional Shale Gas Extraction on Small Streams ». Citizen Science: Theory and Practice, v. 1, n 1, p. 7, 2016.

[ZOO 14] Zooniverse The. 2014. «'citizen science' added to Oxford English Dictionary », Daily Zooniverse, septembre 16, 2014. https://daily.zooniverse.org/2014/09/16/citizen-science-in-dictionary/ 
Annexe 1. Quelques résultats de l'enquête Ipsos de Mai 2016 sur les français et la science participativ

\section{Des craintes qui se renforcent sur la transparence et l'impartialité des scientifiques}

Pour chaque proposition, dites-moi si elle correspond tout à fait, plutôt, plutôt pas ou pas du tout à ce que vous pensez ?

\section{«ST CORRESPOND»}

\begin{abstract}
En France, on peut faire confiance aux scientifiques pour respecter les lois et les règles qui encadrent leurs recherches
\end{abstract}

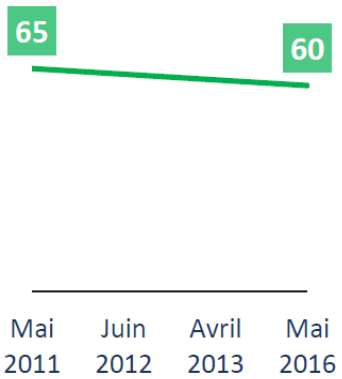

En France, les scientifiques sont aujourd'hui efficacement contrôlés par les autorités de sûreté nationale et européennes

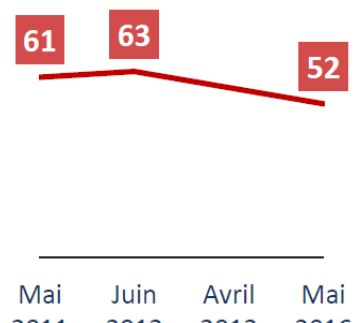

En France, on peut faire confiance aux scientifiques pour dire la vérité si jamais certaines de leurs recherches pouvaient avoir des répercussions sur la santé des individus

$20112012 \quad 2013 \quad 2016$
Les scientifiques français son globalement indépendants et ne se laissent pas influencer par des groupes de pression industriels

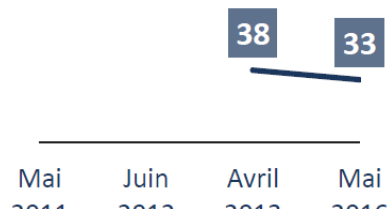

Les sciences participatives sont perçues comme un moyen de renforcer les liens entre le grand public et la recherche et de faire progresser la science

Pensez-vous qu'une implication plus forte du grand public dans les travaux de recherche scientifique pourrait permettre de :

\begin{tabular}{|c|c|c|c|c|}
\hline @ Oui, tout à fait $\quad$ Oui, plutôt & Non, plutôt pas & Non, pas du tout & ST OUI & ST NON \\
\hline $\begin{array}{l}\text { Mieux sensibiliser le grand public sur les questions de préservation de } \\
\text { la biodiversité, la lutte contre le changement climatique, etc. }\end{array}$ & 28 & 103 & $87 \%$ & $13 \%$ \\
\hline $\begin{array}{r}\text { Permettre aux citoyens de mieux comprendre les enjeux de société des } \\
\text { avancées scientifiques et techniques }\end{array}$ & 23 & 112 & $87 \%$ & $13 \%$ \\
\hline Renforcer le lien entre les chercheurs et les citoyens & 23 & 123 & $85 \%$ & $15 \%$ \\
\hline Renforcer l'attractivité des études et des carrières scientifiques & 20 & 154 & $81 \%$ & $19 \%$ \\
\hline $\begin{array}{r}\text { Contribuer à réaliser des avancées majeures en matière de } \\
\text { connaissance scientifique }\end{array}$ & 15 & 174 & $79 \%$ & $21 \%$ \\
\hline
\end{tabular}

La confiance se dégrade dans le champ des recherches climatiques, nucléaires et des OGM

Diriez-vous que vous avez plutôt confiance ou plutôt pas confiance dans les scientifiques pour dire la vérité sur les résultats et les conséquences de leurs travaux dans les domaines suivants... ?

«ST CONFIANCE»

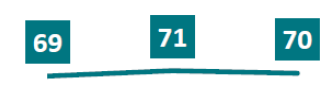

Les énergies nouvelles

Mai 2011 Avril 2013 Mai 2016

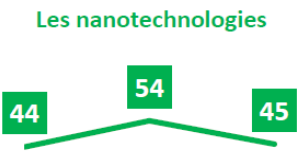

Mai 2011 Avril 2013 Mai 2016

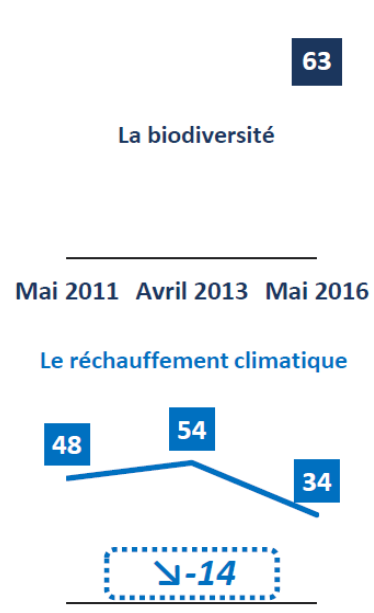

Mai 2011 Avril 2013 Mai 2016
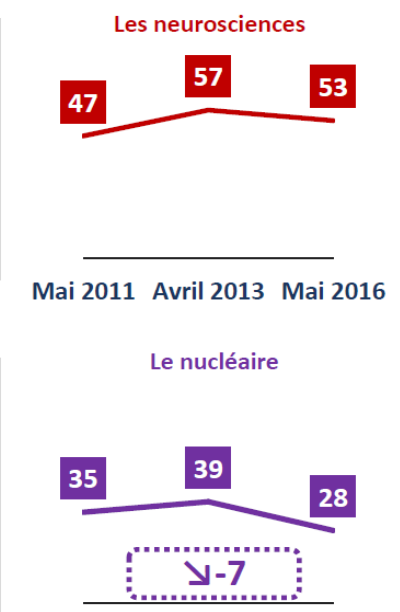

Mai 2011 Avril 2013 Mai 2016

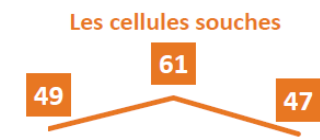

Mai 2011 Avril 2013 Mai 2016

Les OGM

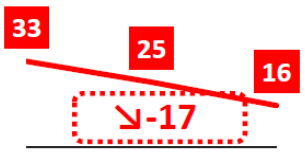

Mai 2011 Avril 2013 Mai 2016

Page | 23 
Annexe 2. Schéma des six étapes d'un scénario idéal relatif à l'utilisation d'un outil web permettant aux participants d'analyser des données

Elaboré par Céline Arnal. Cybelle Planète. 12/2016.

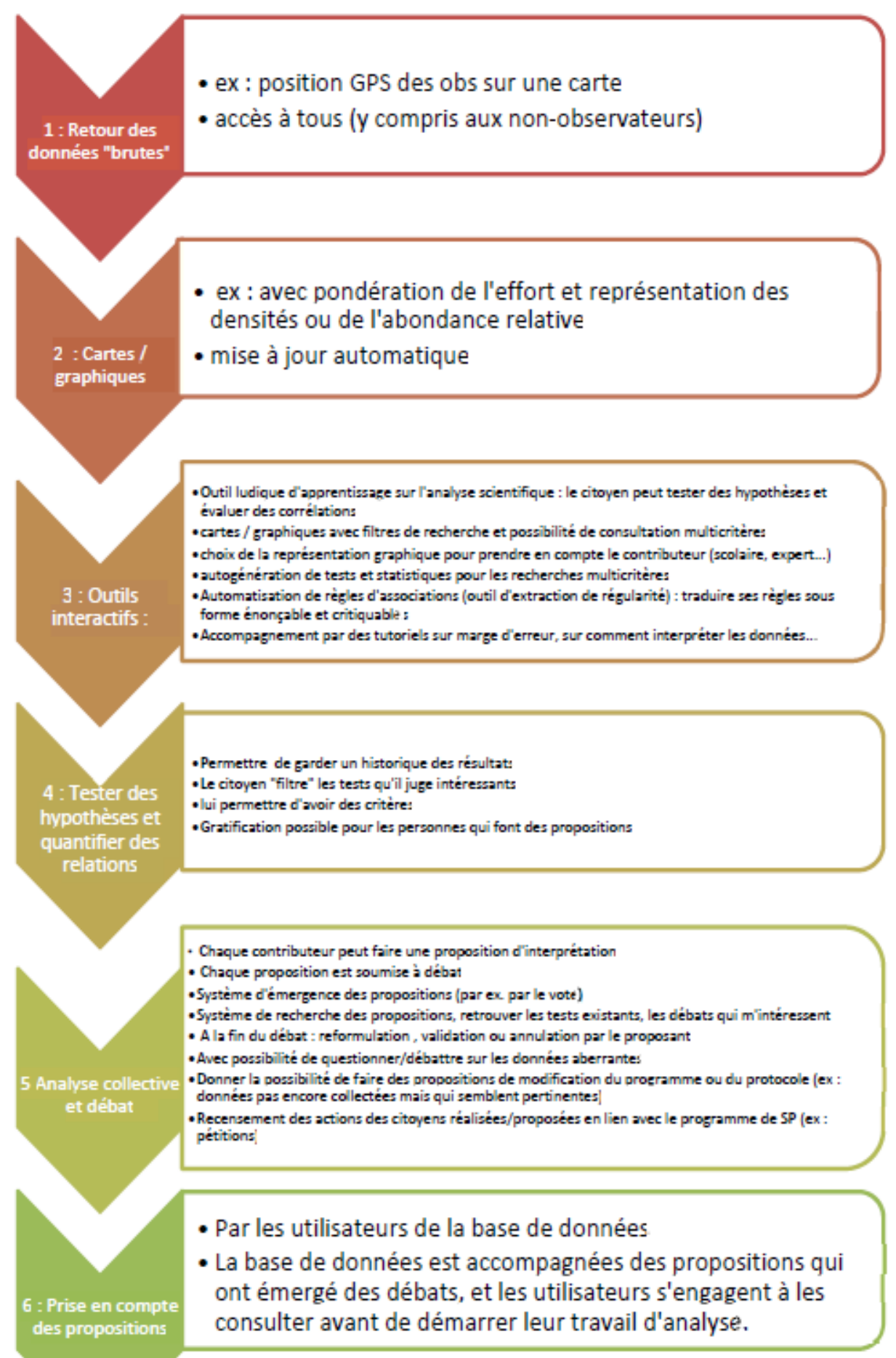


Annexe 3. Mots clefs constitutifs des trois axes de réflexion à l'origine du projet Analyse Citoyenne des Données

\begin{tabular}{|c|c|c|c|}
\hline CITOYENS & RECHERCHE & OUTILS & FONCTIONNALITES \\
\hline $\begin{array}{l}\text { Analyse engagement } \\
\text { des citoyens }\end{array}$ & $\begin{array}{|lr|}\begin{array}{l}\text { Elaboration } \\
\text { question }\end{array} & \text { d'une } \\
\text { recherche } & \text { de } \\
\end{array}$ & $\begin{array}{|lr|}\begin{array}{l}\text { Outils } \\
\text { participatifs }\end{array} & \text { web } \\
\text { collaboratifs } & \text { et } \\
\end{array}$ & $\begin{array}{l}\text { Intégration des } \\
\text { widgets sur le site en } \\
\text { ligne }\end{array}$ \\
\hline $\begin{array}{l}\text { Prise en compte des } \\
\text { citoyens }\end{array}$ & $\begin{array}{l}\text { Publication, } \\
\text { processus } \\
\text { cadrage }\end{array}$ & $\begin{array}{l}\text { Atteindre l'étape } \\
\text { d'un prototype }\end{array}$ & $\begin{array}{l}\text { Outils d'analyse } \\
\text { simple }\end{array}$ \\
\hline $\begin{array}{lr}\begin{array}{l}\text { Identifier } \\
\text { attentes les }\end{array} & \text { les } \\
\text { plus } \\
\text { courantes } \\
\text { citoyens }\end{array}$ & Recherche en ligne & \begin{tabular}{|l} 
Analyse \\
ergonomique
\end{tabular} & $\begin{array}{l}\text { Poser les questions } \\
\text { en co-construction }\end{array}$ \\
\hline $\begin{array}{l}\text { Partage savoir-faire } \\
\text { et connaissances }\end{array}$ & $\begin{array}{l}\text { Produire des } \\
\text { connaissances } \\
\text { écologiques fiables }\end{array}$ & $\begin{array}{l}\text { Un cahier des } \\
\text { charges finalisé }\end{array}$ & $\begin{array}{l}\text { Participatif et débat } \\
\text { démocratique }\end{array}$ \\
\hline $\begin{array}{lr}\text { Identifier } & \text { les } \\
\text { motivations } & \text { des } \\
\text { citoyens } & \text { pour } \\
\text { l'analyse } & \text { des } \\
\text { données } & \end{array}$ & $\begin{array}{lr}\text { Enrichir les données } \\
\text { des } & \text { ateliers } \\
\text { présentiels } & \end{array}$ & $\begin{array}{l}\text { Conception d'une } \\
\text { appli web de } \\
\text { recherche } \\
\text { interactive de } \\
\text { relations de cause à } \\
\text { effet dans les } \\
\text { données de sciences } \\
\text { participatives par les } \\
\text { citoyens }\end{array}$ & $\begin{array}{l}\text { Fonctionnalités } \\
\text { attendues d'un site } \\
\text { participatif }\end{array}$ \\
\hline \begin{tabular}{|ll} 
Répondre & aux \\
besoins & des \\
participants & \\
\end{tabular} & $\begin{array}{l}\text { Poser les questions } \\
\text { en co construction }\end{array}$ & $\begin{array}{l}\text { Cadrage d'un projet } \\
\text { plus ambitieux }\end{array}$ & $\begin{array}{l}\text { Modèle participatif } \\
\text { en virtuel }\end{array}$ \\
\hline $\begin{array}{l}\text { Consultation } \\
\text { citoyennes }\end{array}$ & & $\begin{array}{l}\text { Intégrer les outils } \\
\text { RAP en ligne }\end{array}$ & $\begin{array}{l}\text { Comme Céline l'a } \\
\text { présenté modulo } \\
\text { quelques points }\end{array}$ \\
\hline & & $\begin{array}{l}\text { Co développement } \\
\text { d'outils } \\
\text { exploratoires } \\
\text { (visuels et intuitifs) }\end{array}$ & $\begin{array}{l}\text { Co développement } \\
\text { outils exploration }\end{array}$ \\
\hline $\begin{array}{l}\text { Prise de décisions } \\
\text { collectives }\end{array}$ & & & $\begin{array}{l}\text { Identifier le potentiel } \\
\text { low cost pour des } \\
\text { analyses } \\
\text { collaboratives en } \\
\text { ligne }\end{array}$ \\
\hline $\begin{array}{l}\text { Intégrer le } \\
\text { comportement et la } \\
\text { connaissance des } \\
\text { utilisateurs dans } \\
\text { l'analyse }\end{array}$ & & $\begin{array}{l}\text { Analyse et graphique } \\
\text { compréhensible }\end{array}$ & $\begin{array}{l}\text { Participatif et débat } \\
\text { démocratique }\end{array}$ \\
\hline
\end{tabular}


Annexe 4. Liste des 31 projets étudiés

\begin{tabular}{|c|c|c|c|}
\hline Nom du projet & Lien URL & Domaine & Pays \\
\hline Cochrane Crowd & http://crowd.cochrane.org/index.html & Médecine & International \\
\hline Foldit & https://fold.it/portal/info/about & Biochimie & Etats-Unis \\
\hline Mozak & https://www.mozak.science/game & Neuroscience & Etats-Unis \\
\hline GROW & http://growobservatory.org/ & Agronomie & Angleterre \\
\hline Croprotect & https://croprotect.com/discover & Agronomie & Angleterre \\
\hline Mapping for change & http://mappingforchange.org.uk/ & Multidomaine & Angleterre \\
\hline SENSR & http://www.sensr.org/participateDesc.php & Multidomaine & Etats-Unis \\
\hline ISeeChange & https://www.iseechange.org/ & Environnement & $\begin{array}{l}\text { Etats-Unis } \\
\text { Angleterre }\end{array}$ \\
\hline CyberTracker & http://www.cybertracker.org/ & Environnement & International \\
\hline Nature Notebook & https://www.usanpn.org/nn/connect/project & Environnement & Etats-Unis \\
\hline Snapshot Serengeti & https://www.snapshotserengeti.org/\#/about & Environnement & $\begin{array}{c}\text { International } \\
\text { (Etats-Unis, } \\
\text { Afrique) }\end{array}$ \\
\hline Amazon Aerobotany & $\begin{array}{l}\text { https://www.zooniverse.org/projects/rainfor } \\
\text { estexpeditions/amazon-aerobotany }\end{array}$ & Environnement & Etats-Unis \\
\hline iNaturalist & https://www.inaturalist.org & Environnement & Etats-Unis \\
\hline iSpot & $\begin{array}{l}\text { http://www.ispotnature.org/search/node/Tre } \\
\text { ntepohlia }\end{array}$ & Environnement & Europe \\
\hline Laco Wiki & https://laco-wiki.net/fr/Bienvenue & Environnement & Autriche \\
\hline StallCatchers & https://stallcatchers.com/main & Médecine & Etats-Unis \\
\hline Kaggle & https://www.kaggle.com/competitions & Multidomaine & \\
\hline NOVA Labs & http://www.pbs.org/wgbh/nova/labs/about/ & Multidomaine & Etats-Unis \\
\hline DataONE & https://www.dataone.org/what-dataone & Science de la Vie & Etats-Unis \\
\hline SURVIVOR & $\begin{array}{l}\text { http://www.nancy.inra.fr/Toutes-les- } \\
\text { actualites/Projet-Survivors-Des- } \\
\text { chercheurs-en-herbe }\end{array}$ & Science de la Vie & France \\
\hline PartiCitaE & http://www.particitae.upmc.fr/fr/index.html & Environnement & France \\
\hline E-Bird & http://ebird.org/content/ebird/ & Environnement & Etats-Unis \\
\hline Shale Gas Monitoring & $\begin{array}{l}\text { http://allarmwater.org/cwis438/websites/alla } \\
\text { rm/About.php?WebSitelD=12 }\end{array}$ & Environnement & Etats-Unis \\
\hline Questagame & https://questagame.com/ & Environnement & Australie \\
\hline Creek Watch & http://www.wvwa.org/creekwatch/goals/ & Environnement & Etats-Unis \\
\hline $\begin{array}{l}\text { Shorecomber Trash Blast } \\
\text { Leaders Workshop }\end{array}$ & $\begin{array}{l}\text { http://www.shorecombers.org/workshop- } \\
\text { offerings.html }\end{array}$ & Environnement & \\
\hline Teatime 4 Science & $\begin{array}{l}\text { http://www.teatime4science.org/publication } \\
\underline{\text { s/ }}\end{array}$ & Environnement & Pays-Bas \\
\hline Baby Croinc & https://baby.croinc.org/landing/landing.html & $\begin{array}{l}\text { Développement } \\
\text { humain }\end{array}$ & Israël \\
\hline Climate CoLab & $\begin{array}{l}\text { https://climatecolab.org/page/crowdsourcin } \\
\mathrm{g}\end{array}$ & Environnement & Etats-Unis \\
\hline S'Cool & https://scool.larc.nasa.gov/rover.html & Environnement & Etats-Unis \\
\hline Georgia Adopt-A-Stream & https://aas.gaepd.org/HomePage.aspx & Environnement & Géorgie \\
\hline
\end{tabular}


Annexe 5. Représentation schématique de quelques formes d'analyses rencontrées dans les projets

Nom du projet

\section{Choix de la thématique}

Origine de la donnée

Objectif de l'analyse

Encadrement de l'analyse

Interfaces

Interactions entre communautés
Snapshot Serengeti

\section{iNaturalist}

Degré de liberté faible projet

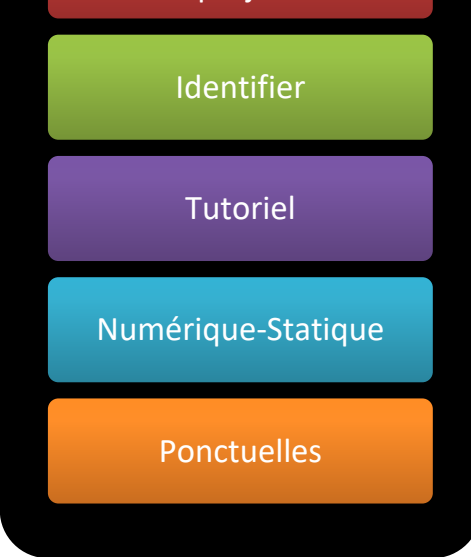

Issue des participants

Kaggle

Degré de liberté important

Issue des participants

Programmer

Identifier - Réviser

Libre

Numérique-Statique

Ponctuelles
Ponctuelles

Libre

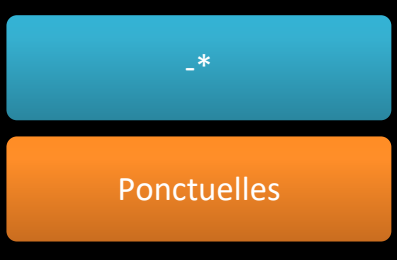

* Nous n'avons pas inscrit d'interface numérique dans le projet Kaggle car l'analyse de données n'a pas lieu sur l'interface numérique. Cette dernière n'est que le lieu de circulation des données analysées par le participant de son côté dans un deuxième temps.

\section{Nom du projet}

\section{Choix de la thématique}

Origine de la donnée

Objectif de l'analyse

Encadrement de l'analyse

Interfaces

Interactions entre communautés
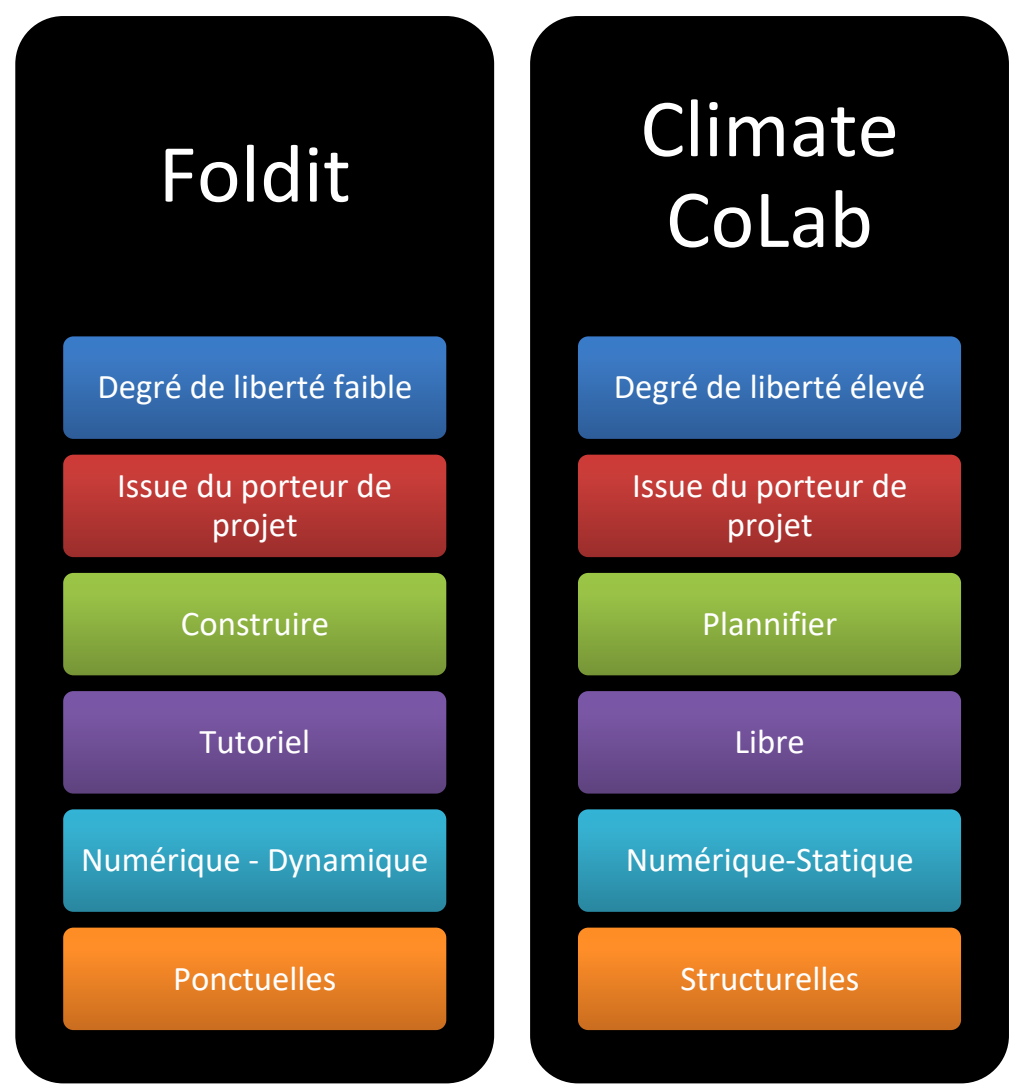